\title{
Measurement Differences, Faults and Instabilities in Intelligent Energy Systems - Part 1: Identification of Overhead High-Voltage Broadband over Power Lines Network Topologies by Applying Topology Identification Methodology (TIM)
}

\author{
Athanasios G. Lazaropoulos* \\ School of Electrical and Computer Engineering, National Technical University of Athens, \\ 9 IroonPolytechniou Street, Zografou, GR 15780 Greece
}

Received August 25, 2016; Accepted September 23, 2016; Published October 3, 2016

\begin{abstract}
This first paper considers the identification of the structure of overhead high-voltage broadband over power lines (OV HV BPL) network topologies by applying the best $\mathrm{L} 1$ Piecewise Monotonic data Approximation (best L1PMA) to measured OV HV BPL transfer functions. Even if measurement differences occur during the determination of an OV HV BPL transfer function, the corresponding OV HV BPL network topology may be revealed through the curve similarity of the best L1PMA result compared with the available records of the proposed OV HV BPL transfer function database.

The contribution of this paper is triple. First, based on the inherent piecewise monotonicity of OV HV BPL transfer functions, best L1PMA is first applied during the determination of theoretical and measured OV HV BPL transfer functions. Second, the creation procedure of the OV HV BPL network topology database is demonstrated as well as the curve similarity performance metric (CSPM). Third, the accuracy of the proposed Topology Identification Methodology (TIM) is examined in comparison with the traditional TIM with respect to the nature of the measurement differences during the determination of OV HV BPL transfer functions.
\end{abstract}

Keywords: Smart Grid; Intelligent Energy Systems; Broadband over Power Lines (BPL) networks; Power Line Communications (PLC); Faults; Fault Analysis; Transmission Power Grids

\section{Introduction}

The deployment of broadband over power lines (BPL) networks across the traditional overhead high-voltage (OV HV) power grid provides a plethora of advantages that render these networks both a useful power grid complement and a strong telecommunications competitor to wireless networking solutions [1]-[4]. Among the characteristics of the emerging power network, some of them that deserve special attention are: (i) its IP-based communications network capabilities; (ii) a great number of smart grid applications; (iii) the low-cost deployment; and (iv) the potential of broadband last mile access through its wired/wireless interfaces due to the power grid ubiquitousness [5]-[13]. 
Nevertheless, when considered as a medium for the transmission of communications signals, OV HV BPL networks are subjected to various inherent deficiencies such as high and frequency-selective channel attenuation and noise [5], [8], [14]-[17]. The broadband potential of OV HV BPL networks is significantly influenced by each of the aforementioned insidious factors [18], [19].

As the channel attenuation is concerned in this paper, the well-established hybrid method, which is employed to examine the behavior of multiconductor transmission line (MTL) structures, is also adopted in this paper [5]-[7], [14]-[17], [20]-[27]. Successfully tested in various transmission and distribution BPL networks [8], [24], [28], the hybrid method is based on: (i) a bottom-up approach that consists of the MTL theory, eigenvalue decomposition (EVD) and singular value decomposition (SVD) [7], [14], [18], [22], [25], [27], [29], [30]; and (ii) a top-down approach that is denoted as TM2 method and is based on the concatenation of multidimensional chain scattering matrices [5]-[7], [14]-[17], [20]-[22], [26], [29]. When the OV HV BPL network topology, OV HV MTL configuration and the applied coupling scheme are given as inputs, the hybrid method gives as an output the corresponding transfer function. In this paper, an OV HV BPL transfer function database is created where all the possible OV HV BPL network topologies are recorded as well as their corresponding transfer functions.

Despite the fact that a plethora of experimental results and theoretical analyses validate the theoretical accuracy of the hybrid method [16], [17], [31], [32], a number of practical reasons and "real-life" conditions may create measurement differences between experimental and theoretical results during the transfer function determination of $\mathrm{OV}$ HVBPL network topologies. On the basis of six measurement difference categories, which are analytically reported in the following analysis, their impact on the transfer functions of transmission BPL networks is evaluated and analyzed. In accordance with [1], to mitigate the aforementioned measurement differences and restore the underlaid theoretical transfer function, best L1PMA has been successfully applied in distribution BPL networks. Among the myriad of data approximation methods that has been proposed in the literature, the application of the best L1PMA, which is theoretically presented and experimentally verified in [33]-[39], is adopted in this paper. Due to its remarkable efficiency to cope with the measurement differences of distribution BPL networks [1], best L1PMA is first applied during the revelation of theoretical OV HV BPL transfer functions. By recognizing the theoretical OV HV BPL transfer function, the respective OV HV BPL network topology can be identified through its curve similarity with the available records of the OV HV BPL transfer function database.

The rest of this paper is organized as follows: In Sec. II, the OV HV MTL configuration and the respective indicative topologies are presented. Sec. III summarizes the principles of BPL signal transmission through the lens of the well-validated hybrid method. The creation procedure of the OV HV BPL transfer function database is also detailed. In Sec.IV, a presentation of the best L1PMA is given. Implementation details, mathematical analysis and the appropriate curve similarity performance metric (CSPM) concerning best L1PMA are analytically reported. Sec.V discusses the simulations of various transmission BPL networks intending to mark out the efficiency of the proposed topology identification methodology (TIM) either to mitigate the occurred measurement differences or to identify OV HV BPL network topologies. Sec.VI concludes this paper. 


\section{Transmission Power Grids}

\subsection{OV HV MTL Configuration}

A typical OV $400 \mathrm{kV}$ double-circuit MTL configuration is depicted in Fig. 1 [6], [8]. This OV HV MTL configuration consists of eight conductors $\left(n^{\text {OVHV }}=8\right)$ that are divided into two categories, namely:

- Phase lines: These lines with radii $r_{\mathrm{p}}^{\mathrm{OVHV}}=15.3 \mathrm{~mm}$ are hung at typical heights $h_{\mathrm{p}}^{\text {OVHV }}$ equal to $20 \mathrm{~m}$ above ground -i.e., conductors $1,2,3,4,5$, and $6-$. The six phase conductors of the OV HV MTL configuration are further divided into three bundles; the phase conductors of each bundle are connected by nonconducting spacers and are separated by $\Delta_{\mathrm{p} 2}^{\mathrm{OVHV}}$ equal to $400 \mathrm{~mm}$, whereas bundles are spaced by $\Delta_{\mathrm{p} 1}^{\mathrm{OVHV}}$ equal to $10 \mathrm{~m}$.

- Neutral conductors: Except for the phase conductors, two parallel neutral conductors, which are the conductors 7 and 8 , are hung at heights $h_{\mathrm{n}}^{\text {OVHV }}$ equal to $23.7 \mathrm{~m}$. The neutral conductors with radii $r_{\mathrm{n}}^{\text {OVHV }}=9 \mathrm{~mm}$ are spaced by $\Delta_{\mathrm{n}}^{\text {OVHV }}$ equal to $12 \mathrm{~m}$. 


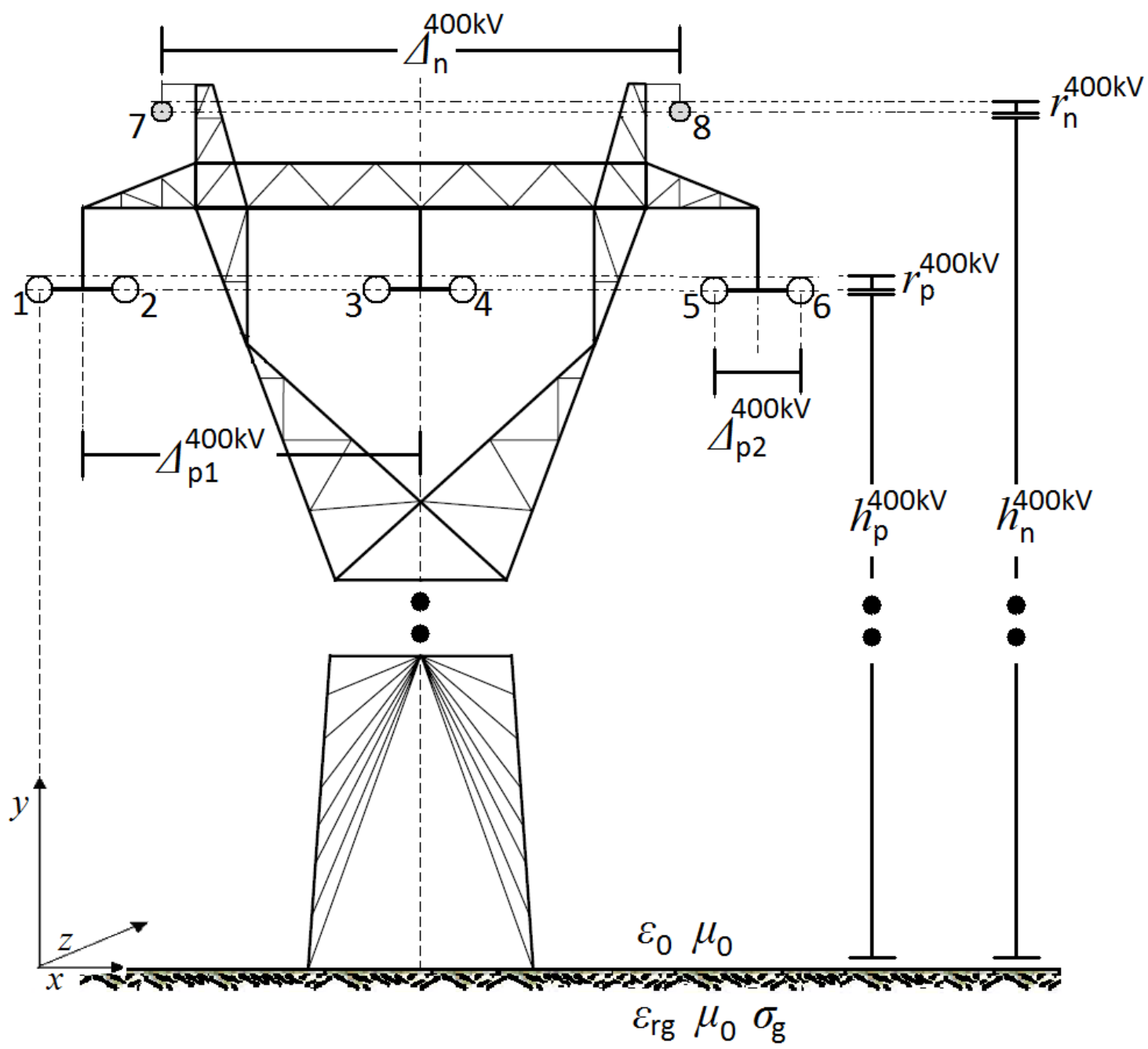

Figure 1.OV 400kV double-circuit MTL configuration [6], [8].

In accordance with [5], [6], [8], [40], [41], the ground with conductivity $\sigma_{\mathrm{g}}=5 \mathrm{mS} / \mathrm{m}$ and relative permittivity $\varepsilon_{\mathrm{rg}}=13$ is considered as the reference conductor. The aforementioned ground parameters define a realistic scenario during the following analysis while the impact of imperfect ground on broadband signal propagation via OV HV power lines was analyzed in [5], [6], [14]-[17], [21], [25], [40], [42], [43].

\subsection{Indicative OV HV BPL Topologies}

Since OV HV BPL networks suffer from various inherent deficiencies such as high and frequency-selective channel attenuation and noise, BPL networks are divided into cascaded BPL topologies in order to cope with these deficiencies [1], [5], [8], [14]-[17], [44], [45]. Each OV HV BPL topology, which is denoted as network module, is bounded by the transmitting and receiving end where respective BPL repeaters are installed. The role of BPL repeaters is to extract the attenuated BPL signal, amplify it while improving its signal-to-noise ratio and inject it in OV HV lines. Between the two BPL repeaters of each OV HV BPL topology, $N$ successive branches are encountered. 
According to the previous description, a general OV HV BPL topology is presented in Fig. 2.

In accordance with [5]-[7], [14]-[17], [20]-[26], [29], [40], [46], [47] and with reference to Fig. 2, average path lengths of the order of $25 \mathrm{~km}$ are considered in OV HV BPL topologies. The following three indicative OV HV BPL topologies, concerning end-to-end connections of average path lengths, are examined, namely [5], [6]:

1. A typical suburban topology (OV HV suburban case) with $N=2$ branches $\left(L_{1}=9 \mathrm{~km}\right.$, $\left.L_{2}=13 \mathrm{~km}, L_{3}=3 \mathrm{~km}, L_{\mathrm{b} 1}=17 \mathrm{~km}, L_{\mathrm{b} 2}=13 \mathrm{~km}\right)$.

2. A typical rural topology (OV HV rural case) with only $N=1$ branch $\left(L_{1}=4 \mathrm{~km}, L_{2}=21 \mathrm{~km}, L_{\mathrm{b} 1}=24 \mathrm{~km}\right)$.

3. The "LOS" transmission along the same end-to-end distance $L=L_{1}+\ldots+L_{N+1}=25 \mathrm{~km}$ when no branches are encountered. This topology corresponds to Line of Sight transmission in wireless channels.

The three indicative OV HV BPL topologies are going to be used so that the accuracy of the proposed TIM is evaluated in Sec.V.

As the circuital parameters of OV HV BPL topologies are regarded [5]-[7], [14]-[17], [20]-[26], [29], [40], [41], [46], [47], several assumptions need to be taken into account: (i) The branching cables are assumed identical to the transmission cables; (ii) The interconnections between the transmission and branch conductors are fully activated; (iii) The transmitting and the receiving ends are assumed matched to the characteristic impedance of the modal channels; and (iv) The branch terminations are assumed open circuits.

\subsection{OV HV BPL Topologies of the Proposed Database}

As it has already been mentioned in Sec.IIB, average path lengths $L$ of the order of $25 \mathrm{~km}$ are considered in OV HV BPL topologies. To create a detailed OV HV BPL topology database, all the possible OV HV BPL topologies concerning the number of branches, branch distance from the transmitting end and branch length need to be inserted in the database. In order to simplify the following analysis without harming the generality of the proposed TIM, the maximum number of branches, the length spacing for both branch distance and branch length and the maximum branch length are assumed to be equal to $N, L_{\mathrm{s}}$ in $\mathrm{km}$ and $L_{\mathrm{b}}$ in $\mathrm{km}$, respectively. Therefore, the following cases are reported for each number of branches with reference to Fig. 2:

- 1 topology when no branches are encountered (i.e., "LOS" transmission case).

- When one branch is assumed, there are $L / L_{\mathrm{s}}+1$ possible branch locations for the length $L_{1}$ across the "LOS" transmission path. Here, it should be noted that the branch may also be located either at the transmitting $\left(L_{1}=0 \mathrm{~km}\right)$ or at the receiving end $\left(L_{1}=L\right)$. If the $L_{\mathrm{b}} / L_{\mathrm{s}}+1$ possible branch lengths of the length $L_{\mathrm{b} 1}$ are considered then $\left(\frac{L}{L_{\mathrm{s}}}+1\right) \times\left(\frac{L_{\mathrm{b}}}{L_{\mathrm{s}}}+1\right)$ topologies of one branch should be inserted in the OV HV BPL topology database.

- When two branches are assumed, let the first branch be located at distance $L_{1}$ from the transmitting end. To avoid duplicates in the OV HV BPL topology database, the second branch can be located at distance $L_{2}$ from the first branch 


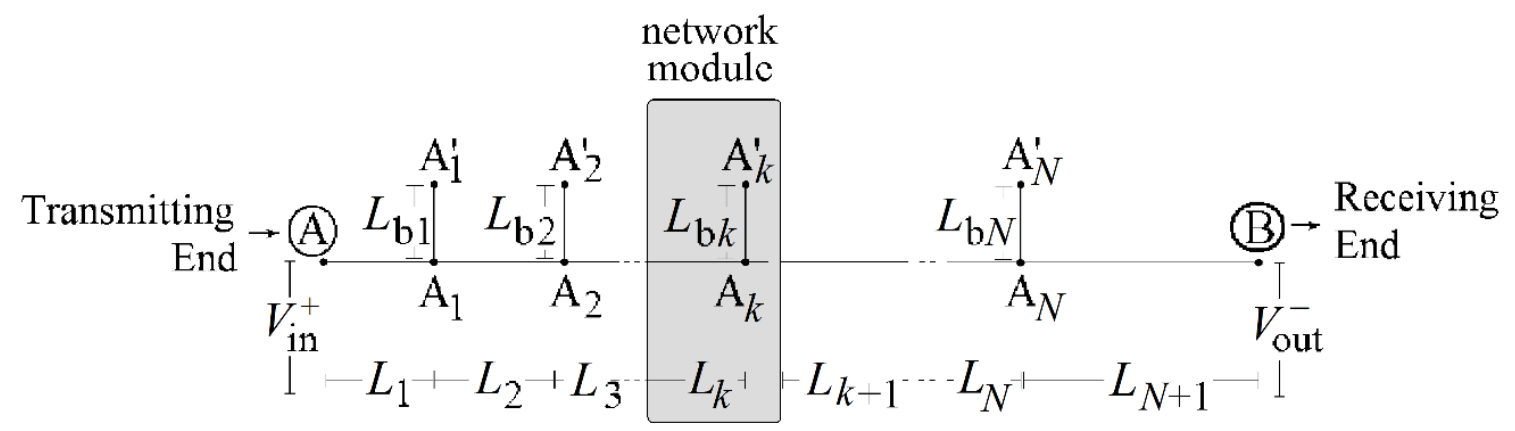

Figure 2. General OV HV BPL topology [6], [8].

that varies from 0 (double branch) to $L-L_{1}$. Since the average path length is assumed equal to $L$ in all the cases examined, the length $L_{3}$ is equal to $L-L_{1}-L_{2}$. If the branch length $L_{\mathrm{b} 1}$ and $L_{\mathrm{b} 2}$ are also taken into account then the number of possible OV HV BPL topologies, which is inserted in the OV HV BPL topology database, is equal to $\sum_{i_{1}=1}^{L / L_{\mathrm{s}}+1} i \times\left(\frac{L_{\mathrm{b}}}{L_{\mathrm{s}}}+1\right)^{2}$.

- Similarly to the two branch case, when $N$ branches are considered across the "LOS" transmission path, there are

$$
\sum_{i_{1}=1}^{L / L_{\mathrm{s}}+1}\left\{\sum_{i_{2}=1}^{i_{1}}\left\{\cdots \sum_{i_{N-1}=1}^{i_{N-2}}\left\{i_{N-1}\right\}\right\}\right\} \times\left(\frac{L_{\mathrm{b}}}{L_{\mathrm{s}}}+1\right)^{N}
$$

possible OV HV BPL topologies that should be inserted in the OV HV BPL topology database.

Since all the possible OV HV BPL topologies are inserted in the database as separate records, each record is accompanied by: (i) its theoretical OV HV BPL transfer function by applying the hybrid method, whose evaluation is detailed in Sec.IIIB and IIIC; and (ii) its approximated OV HV BPL transfer functions by applying the best L1PMA, whose evaluation is detailed in Sec.IVC.

\section{A Briefing of BPL Propagation and Transmission Analysis}

\subsection{MTL Theory}

As it has already been mentioned in [5]-[7], [14]-[17], [20]-[22], [25], [40], [44], through a matrix approach, the standard TL analysis can be extended to the MTL case. In the traditional case of a two-conductor line, one forward- and one backward-traveling wave are supported. However, in the MTL case where the MTL structure consists of $n^{\mathrm{OVHV}}+1$ conductors parallel to the $\mathrm{z}$ axis (see Fig. 1), $n^{\mathrm{OVHV}}$ pairs of forward- and backward-traveling waves with corresponding propagation constants may be supported. Each pair of forward- and backward-traveling waves is referred to as a mode and their OV HV spectral behavior has already been investigated in [5]-[7], [14]-[17], [20]-[22], [25]-[27], [29], [30], [40]-[44], [47], [48]. 


\subsection{EVD Modal Analysis and Channel Transfer Functions}

With reference to Fig.1, the $n^{\mathrm{OVHV}}$ modes, which are supported by the examined

MTL configuration, may propagate across OV HV BPL topologies. Through TM2 method, which is based on the scattering matrix theory and presented analytically in [7], [22], their spectral behavior is described by the $n^{\mathrm{OVHV}} \times n^{\mathrm{OVHV}} \mathrm{EVD}$ modal transfer function matrix $\mathbf{H}^{\mathrm{m}}\{\}$ whose elements $H_{i, j}^{\mathrm{m}}\left\{\{\}, i, j=1, \ldots, n^{\text {ovHV }}\right.$ are the EVD modal transfer functions where $H_{i, j}^{\mathrm{m}}$ denotes the element of matrix $\left.\mathbf{H}^{\mathrm{m}}\{\}\right\}$ in row $i$ of column $j$. Through appropriate similarity transformations, which are presented in [5]-[7], [14]-[17], [20]-[22], [25], [40], [44], the EVD modal transfer functions are related with the line transfer functions through the $n^{\mathrm{OVHV}} \times n^{\mathrm{OVHV}}$ channel transfer function matrix $\mathbf{H}\{\}$ that is determined from

$$
\mathbf{H}\{\cdot\}=\mathbf{T}_{V} \cdot \mathbf{H}^{m}\{\cdot\} \cdot \mathbf{T}_{V}^{-1}
$$

where $\mathbf{T}_{V}$ is a $n^{\mathrm{OVHV}} \times n^{\mathrm{OVHV}}$ matrix depending on the frequency, the MTL configuration and the physical properties of the cables.

\subsection{Coupling Schemes and Coupling Transfer Functions}

According to how signals are injected into OV HV lines, two coupling schemes are mainly supported by the OV HV BPL networks, as folows [6], [7], [11], [12], [28]:

- Wire-to-Ground (WtG) when the signal is injected into one conductor and returns via the ground; say between conductor $s, s=1, \ldots, 8$ and the ground. The coupling WtG channel transfer function $\left.H^{\mathrm{WtG}}\{\}\right\}$ is given from

$$
H^{\mathrm{WtG}}\{\cdot\}=\left[\mathbf{C}^{\mathrm{WtG}}\right]^{\mathrm{T}} \cdot \mathbf{T}_{V} \cdot \mathbf{H}^{m}\{\cdot\} \cdot \mathbf{T}_{V}^{-1} \cdot \mathbf{C}^{\mathrm{WtG}}
$$

where $\mathbf{C}^{\mathrm{WtG}}$ is an $8 \times 1$ coupling column vector with zero elements except in row $s$ where the value is equal to 1 . WtG coupling between conductor $s$ and ground will be detoned as $\mathrm{WtG}^{s}$, hereafter.

- Wire-to-Wire $(\mathrm{WtW})$ when the signal is injected between two conductors; say between conductors $p$ and $q \neq p, p, q=1, \ldots, 8$. Similarly to eq. (3), the coupling $\mathrm{WtW}$ channel transfer function $H^{\mathrm{Wtw}}\{$.$\} is given from$

$$
H^{\mathrm{Wtw}}\{\cdot\}=\left[\mathbf{C}^{\mathrm{WtW} \text {,ut }}\right]^{\mathrm{T}} \cdot \mathbf{T}_{V} \cdot \mathbf{H}^{m}\{\cdot\} \cdot \mathbf{T}_{V}^{-1} \cdot \mathbf{C}^{\mathrm{WtW}, \text { in }}
$$

where $\mathbf{C}^{\mathrm{WtW} \text {,ut }}$ and $\mathbf{C}^{\mathrm{WtW} \text {;n }}$ are $8 \times 1$ output and input coupling column vectors, respectively.

Since the main interest of this paper is the accuracy evaluation of the proposed TIM, only one of the previous coupling schemes is going to be applied in the following analysis for the sake of clarity and terseness; say, WtG coupling schemes.

\section{Best L1PMA}

\subsection{Best L1PMA and BPL Networks}

Among the various proposed monotonic data approximation methods, the application of the best L1PMA, which is theoretically presented and experimentally verified in [33]-[38], successfully copes with problems that are derived from the 
univariate signal restoration such as those presented during the BPL transfer function determination. Until now, the efficacy of best L1PMA to mitigate measurement differences during the determination of distribution BPL transfer functions has already been verified in [1]. In this paper, best L1PMA is first applied to OV HV BPL transfer functions either to mitigate the occurred measurement differences or to identify OV HV BPL topologies.

\subsection{Implementation Details}

Contrary to spline and wavelet approximation methods [49], [50], best L1PMA avoids the assumption that BPL transfer functions depend on certain critical parameters. Actually, the smoothing process is a straightforward data projection when best L1PMA is adopted [1], [34], [35].

Best L1PMA exploits the piecewise monotonicity property that always occurs in transmission and distribution BPL transfer functions [1]. Best L1PMA decomposes BPL transfer functions into separate monotonous sections between adjacent turning points (primary extrema) of the examined transfer functions [35], [36]. By identifying the separate monotonous sections, best L1PMA separately handles them, thus, achieving very good results especially concerning the mitigation of uncorrelated measurement differences [1], [51]. Since best L1PMA is based on the minimization of the moduli sum of the measurement differences, the existence of few large measurement differences among the data make no difference to the best fit [51]. Therefore, the undulation of the OV HV BPL coupling transfer function data due to the existence of deep spectral notches and other measurement differences can be comfortably accommodated through the application of the best L1PMA.

Apart from its easy theoretical implementation concept, another basic advantage of the best L1PMA is its software availability and comprehensibility. The Fortran software package that is applied to implement the best L1PMA has extensively been verified in various scientific fields [36], [38], [52]-[54] and is freely available online in [39]. As the easiness plays critical role, based on the best L1PMA software specifications of [39], best L1PMA receives as inputs the measured OV HV BPL coupling transfer function, the measurement frequencies, the number of monotonic sections (i.e., either user- or computer-defined) and the type of the first monotonic section (i.e., either increasing or decreasing). Then, by executing the Fortran software package, which is a straightforward procedure, the outputs of best L1PMA are the optimal primary extrema and the best fit of the measured OV HV BPL coupling transfer function.

\subsection{The Nature of Measurement Differences and Best L1PMA Mathematical Analysis}

As already been mentioned, a set of practical reasons and "real-life" conditions create significant differences between experimental measurements and theoretical results during the transfer function determination of BPL networks. The reasons for these measurement differences can be grouped into six categories, namely [1], [55]-[57]: (i) Isolation difficulties of specific MTL parameters in time- and frequency-domain; (ii) Low accuracy and sensitivity of the used equipment during measurements; (iii) Cross-talk and resonant phenomena due to the parasitic capacitances and inductances of OV HV lines; (iv) The weakness of including specific wiring and grounding practices; 
(v) Practical impedance deviations of $\mathrm{OV} \mathrm{HV}$ lines, branches, terminations and transmitting/receiving ends; and (vi) The isolation lack of the noise effect during the transfer function computations. Taking under consideration the six measurement difference categories and eq. (4), the measured OV HV coupling transfer function $\overline{H^{\mathrm{WtG}}}\{\cdot\}$ is then determined by

$$
\overline{H^{\mathrm{WtG}}}\left(f_{i}\right)=H^{\mathrm{WtG}}\left(f_{i}\right)+e\left(f_{i}\right)
$$

where $f_{i}$ denotes the measurement frequency and $e\left(f_{i}\right)$ synopsizes the total measurement difference due to the aforementioned six categories.

Generalizing eq. (5), the theoretical OV HV coupling transfer functions $H^{\mathrm{WtG}}\left(f_{i}\right), i=1, \ldots, u$ with respect to measurement frequencies $f_{i}, i=1, \ldots, u$ are regarded as elements of $\mathbf{H}^{\mathrm{WtG}}$ where

$$
\mathbf{H}^{\mathrm{WtG}} \equiv \mathbf{H}^{\mathrm{WtG}}(\mathbf{f})=\left[\begin{array}{lllll}
H^{\mathrm{WtG}}\left(f_{1}\right) & \cdots & H^{\mathrm{WtG}}\left(f_{i}\right) & \cdots & H^{\mathrm{WtG}}\left(f_{u}\right)
\end{array}\right]^{\mathrm{T}}
$$

is the $u \times 1$ theoretical $\mathrm{OV} \mathrm{HV}$ coupling transfer function column vector and $\mathbf{f}=\left[\begin{array}{lllll}f_{1} & \cdots & f_{i} & \cdots & f_{u}\end{array}\right]^{\mathrm{T}}$ is the $u \times 1$ measurement frequency column vector. Similarly, the measured OV HV coupling transfer functions $\overline{H^{\mathrm{WtG}}}\left(f_{i}\right), i=1, \ldots, u$ with respect to measurement frequencies $f_{i}, i=1, \ldots, u$ are regarded as elements of $\overline{\mathbf{H}^{\mathrm{WtG}}}$ where

$$
\overline{\mathbf{H}^{\mathrm{WtG}}} \equiv \overline{\mathbf{H}^{\mathrm{WtG}}}(\mathbf{f})=\left[\begin{array}{lllll}
\overline{H^{\mathrm{WtG}}}\left(f_{1}\right) & \cdots & \overline{H^{\mathrm{WtG}}}\left(f_{i}\right) & \cdots & \overline{H^{\mathrm{WtG}}}\left(f_{u}\right)
\end{array}\right]^{\mathrm{T}}
$$

is the $u \times 1$ measured $\mathrm{OV} \mathrm{HV}$ coupling transfer function column vector. Thus, with reference to Sec.IIIC, best L1PMA receives as inputs the measured OV HV coupling transfer function column vector $\overline{\mathbf{H}^{\mathrm{WtG}}}(\mathbf{f})$, the measurement frequencies $\mathbf{f}$, the number of monotonic sections $k_{\text {sect }}$ and the type of the first monotonic section while best L1PMA gives as outputs the optimal primary extrema and the approximated OV HV coupling transfer function column vector $\overline{\overline{\mathbf{H}_{\text {meas }}^{\mathrm{WtG}}}}\left(\mathbf{f}, k_{\text {sect }}\right)$ by minimizing the sum of the absolute errors

$$
\operatorname{Er}\left(\overline{\mathbf{H}^{\mathrm{WtG}}}, k_{\text {sect }}\right)=\sum_{i=1}^{u} \mid \overline{\mathbf{H}_{\text {meas }}^{\mathrm{WtG}}}\left(f_{i}, k_{\text {sect }}\right)-\overline{\mathbf{H}^{\mathrm{WtG}}}\left(f_{i}\right)
$$

subject to the piecewise monotonicity constraints

$$
\begin{aligned}
& \overline{\overline{\mathbf{H}_{\text {meas }}^{\mathrm{WtG}}}}\left(f_{\phi_{j-1}}, k_{\text {sect }}\right) \leq \overline{\overline{\mathbf{H}_{\text {meas }}^{\mathrm{WtG}}}}\left(f_{\phi_{j-1}+1}, k_{\text {sect }}\right) \leq \cdots \leq \overline{\overline{\mathbf{H}_{\text {meas }}^{\mathrm{WtG}}}}\left(f_{\phi_{j}}, k_{\text {sect }}\right), \text { if } j \text { is odd } \\
& \left.\overline{\overline{\mathbf{H}_{\text {meas }}^{\mathrm{WtG}}}}\left(f_{\phi_{j-1}}, k_{\text {sect }}\right) \geq \overline{\overline{\mathbf{H}_{\text {meas }}^{\mathrm{WtG}}}}\left(f_{\phi_{j-1}+1}, k_{\text {sect }}\right) \geq \cdots \geq \overline{\overline{\mathbf{H}_{\text {meas }}^{\mathrm{WtG}}}}\left(f_{\phi_{j}}, k_{\text {sect }}\right) \text {, if } j \text { is even }\right\}
\end{aligned}
$$

where the integer numbers $\phi_{j}, j=1, \ldots, k_{\text {sect }}$ define the positions of the primary extrema of the best L1PMA satisfying the conditions

$$
1=\phi_{0} \leq \phi_{1} \leq \cdots \leq \phi_{k_{\text {sect }}}=u
$$

\subsection{Best L1PMA and Theoretical OV HV Coupling Transfer Functions}

Apart from its theoretical OV HV BPL coupling transfer function, each OV HV BPL topology is accompanied with a number of approximated theoretical OV HV BPL coupling transfer functions that come from the application of the best L1PMA for different monotonic sections. With reference to eqs. (8)-(10), the approximated 
theoretical OV HV BPL coupling transfer function column vector $\overline{\overline{\mathbf{H}_{\text {theor }}^{\mathrm{WtG}}}}\left(\mathbf{f}, k_{\text {sect }}\right)$ is the output of the best L1PMA when the measured OV HV BPL coupling transfer function column vector $\overline{\mathbf{H}^{\mathrm{WtG}}}(\mathbf{f})$ is replaced by the theoretical one $\mathbf{H}^{\mathrm{WtG}}(\mathbf{f})$ for given number of monotonic sections.

\subsection{Traditional TIM}

The traditional TIM is rather a rule of thumb than an accurate description. The traditional TIM is based on the minimization of the sum of the absolute errors between measured $\mathrm{OV} \mathrm{HV}$ coupling transfer function column vector $\overline{\mathbf{H}^{\mathrm{WtG}}}(\mathbf{f})$ and theoretical OV HV coupling transfer function column vector $\mathbf{H}^{\mathrm{WtG}}(\mathbf{f})$, say

$$
\operatorname{Er}\left(\overline{\mathbf{H}^{\mathrm{WtG}}}, \mathbf{H}^{\mathrm{WtG}}(\mathbf{f})\right)=\sum_{i=1}^{u} \mid \overline{\mathbf{H}^{\mathrm{WtG}}}\left(f_{i}\right)-\mathbf{H}^{\mathrm{WtG}}\left(f_{i}\right)
$$

and the belief that the underlaid OV HV BPL topology is the one that presents the minimum sum of eq. (11). However, this belief is far away the truth since the decision becomes more wrong as the maximum magnitude of measurement differences increases. Even if small measurement difference magnitudes are assumed, the OV HV BPL topology of the database that presents the minimum sum of eq. (11) rarely is the desirable one (see Sec.VE). In contrast, a successful TIM is the one that could give either the exact OV HV BPL topology, which is difficult due to the high number of OV HV BPL topologies of the database and different difference measurement magnitudes, or a set of candidate OV HV BPL topologies. Indeed, the proposed identification method offers a set of candidate OV HV BPL topologies where the desired OV HV BPL topology lays among them.

\subsection{CSPM and the Proposed TIM}

With reference to eqs. (8)-(10), best L1PMA gives the approximated OV HV coupling transfer function column vector $\overline{\overline{\mathbf{H}_{\text {meas }}^{\mathrm{WtG}}}}\left(\mathbf{f}, k_{\text {sect }}\right)$ when measured OV HV one $\overline{\mathbf{H}^{\mathrm{WtG}}}(\mathbf{f})$ and a number of monotonic sections $k_{\text {sect }}$ are considered. The performance metric of the sum of the absolute errors, which is presented in eq. (8) and is, hereafter, denoted as CSPM, is also applied for the assessment of the curve similarity between the best L1PMA measurement approximation and theory, namely:

$$
C S P M_{k_{\text {sect }}} \equiv C S P M_{k_{\text {sect }}}\left(\overline{\mathbf{H}^{\mathrm{WtG}}}, k_{\text {sect }}\right)=\sum_{i=1}^{u}\left|\overline{\overline{\mathbf{H}_{\text {meas }}^{\mathrm{WtG}}}}\left(f_{i}, k_{\text {sect }}\right)-\overline{\overline{\mathbf{H}_{\text {theor }}^{\mathrm{WtG}}}}\left(f_{i}, k_{\text {sect }}\right)\right|
$$

The proposed TIM is based on the CSPM and the OV HV topology database in order to identify the OV HV BPL topology when a set of coupling transfer function measurements is available. Actually, the methodology follows the following three steps so that the OV HV BPL topology is revealed:

1. Given the measured OV HV coupling transfer function column vector $\overline{\mathbf{H}^{\mathrm{WtG}}}(\mathbf{f})$, the approximated OV HV coupling transfer function column vector $\overline{\overline{\mathbf{H}_{\text {meas }}^{\mathrm{WtG}}}}\left(\mathbf{f}, k_{\text {sect }}\right)$ 
is evaluated for monotonic sections that range from 1 to $k_{\text {sect,max }}$ where $k_{\text {sect,max }}$ is the maximum number of monotonic sections considered.

2. For each OV HV BPL topology and each monotonic section of the OV HV BPL topology database, the respective $C S P M k_{\text {sect }}$ of eq. (12) is computed. Then, for each OV HV BPL topology, the total CSPM tot is determined from

$$
C S P M_{\text {tot }} \equiv \sum_{k_{\text {sect }}=1}^{k_{\text {sect, max }}} C S P M_{k_{\text {sect }}}
$$

3. A set of candidate OV HV BPL topologies with their respective CSPM tot is provided by the TIM. This set of candidates OV HV BPL topologies is characterized by the lowest CSPM tot among all the available OV HV BPL topologies of the database. The number of candidate OV HV BPL topologies depends on the topological characteristics of the underlaid topology (i.e., number of branches, branch length), the nature of measurement differences (i.e., measurement difference distributions, characteristics of distributions) and the number of monotonic sections applied.

As it is evident from the previous steps, CSPM acts either as a curve similarity metric or as a topology identification tool. Finally, the simulation results of the proposed TIM are shown in Sec.VE.

\section{Numerical Results and Discussion}

\subsection{Simulation Goals and Parameters}

Various types of transmission BPL networks are simulated with the purpose of evaluating the proposed TIM. More specifically, the efficiency of the methodology is assessed with reference to a number of factors, such as the transmission BPL topology and the nature of measurement differences.

As regards the hybrid method specifications, the BPL frequency range and the flat-fading subchannel frequency spacing are assumed equal to $1-30 \mathrm{MHz}$ and $1 \mathrm{MHz}$, respectively. Therefore, the number of subchannels $u$ in the examined frequency range is equal to 30. Arbitrarily, the $\mathrm{WtG}^{3}$ coupling scheme is applied during the following simulations. As it is usually done [5], [20]-[23], [58], the selection of representative coupling schemes is a typical procedure for the sake of reducing manuscript size and simplicity.

As the OV HV BPL topology database specifications are concerned, the maximum number of branches $N$, the length spacing $L_{\mathrm{s}}$ for both branch distance and branch length and the maximum branch length $L_{\mathrm{b}}$ are assumed equal to $3,1 \mathrm{~km}$ and $25 \mathrm{~km}$, respectively.

Finally, the maximum number of monotonic sections $k_{\text {sect,max }}$ that is going to be used during the application of best L1PMA is assumed to be equal to 20 [1].

\subsection{Theoretical and Approximated OV HV BPL Transfer Functions}

Prior to understanding the proposed TIM, there is a need for recognizing: (i) how best L1PMA approximates theoretical coupling transfer functions of transmission OV BPL topologies, such those presented in Sec.IIB, and (ii) the role of the number of the monotonic sections during the OV HV BPL coupling transfer function approximation. 
In Figs. 3(a)-(c), the theoretical coupling transfer function is plotted versus frequency for the three indicative OV HV BPL topologies of the Sec.IIB, respectively. In each figure, the best L1PMA result is also plotted for a number of representative monotonic sections (i.e., $k_{\text {sect }}=2, k_{\text {sect }}=5$ and $k_{\text {sect }}=20$ ). In Table 1 , the average error between theoretical coupling transfer function and the respective best L1PMA output is reported for different number of monotonic sections when the three indicative OV HV BPL topologies are considered. 


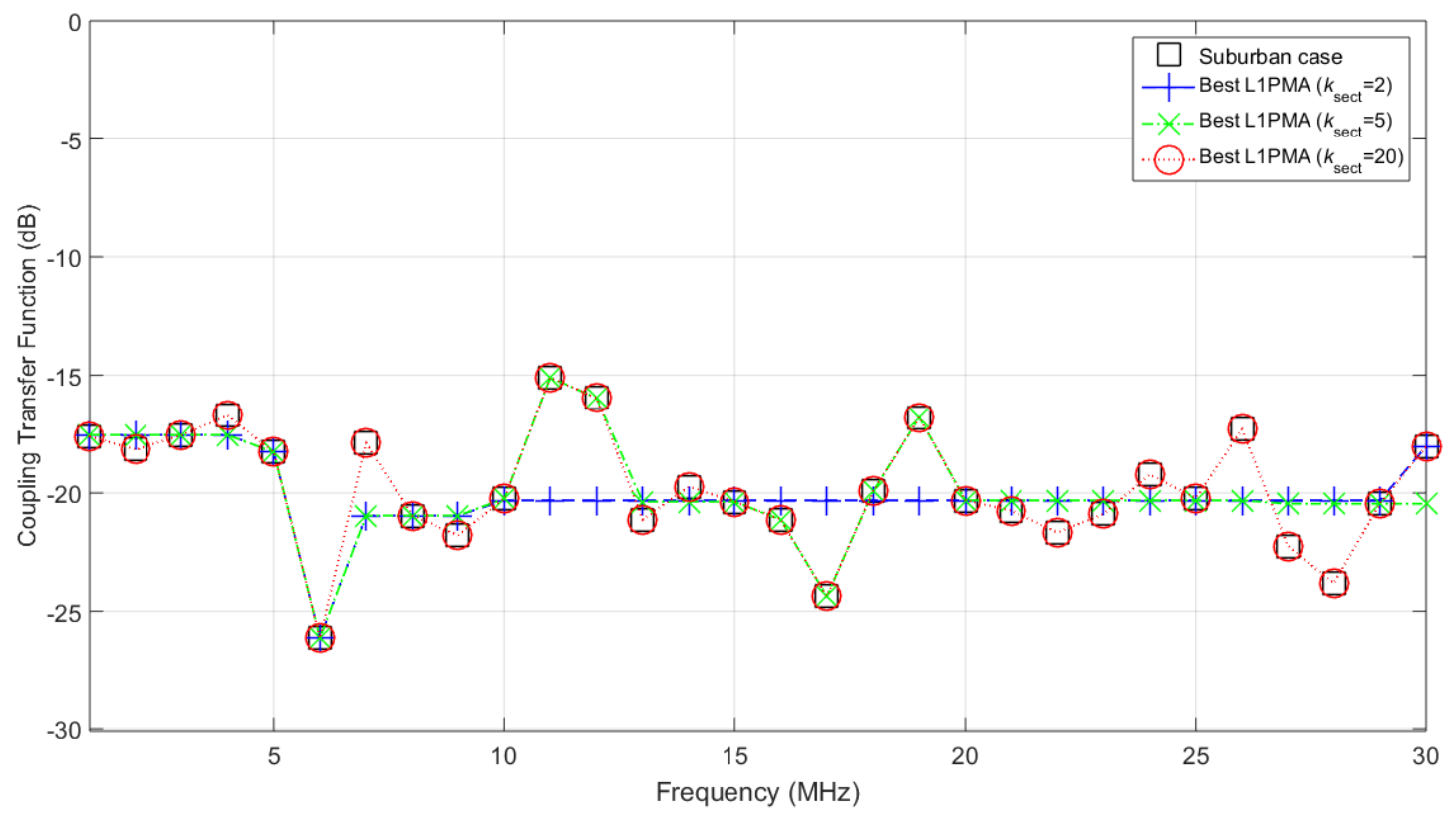

(a)

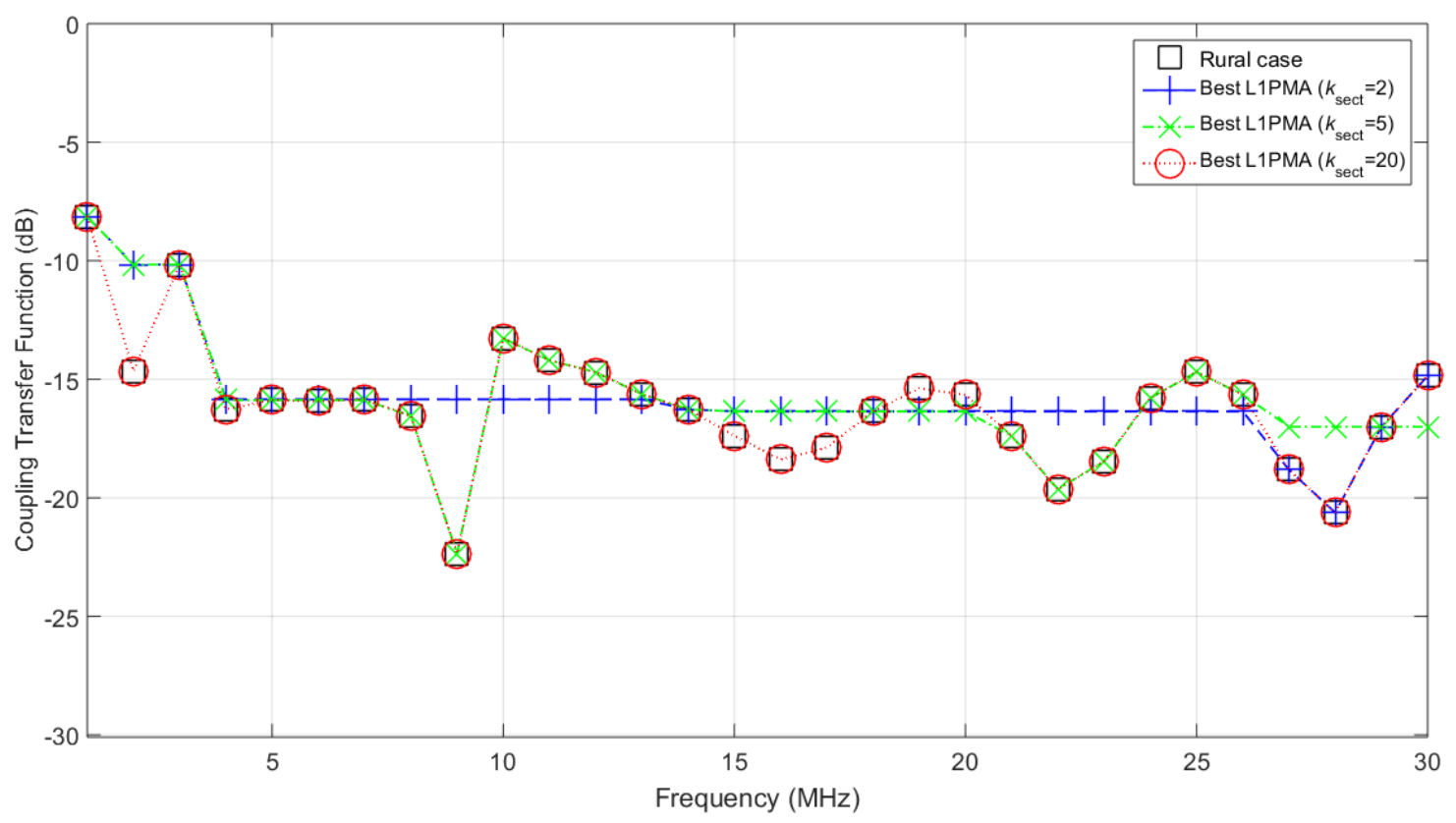

(b) 


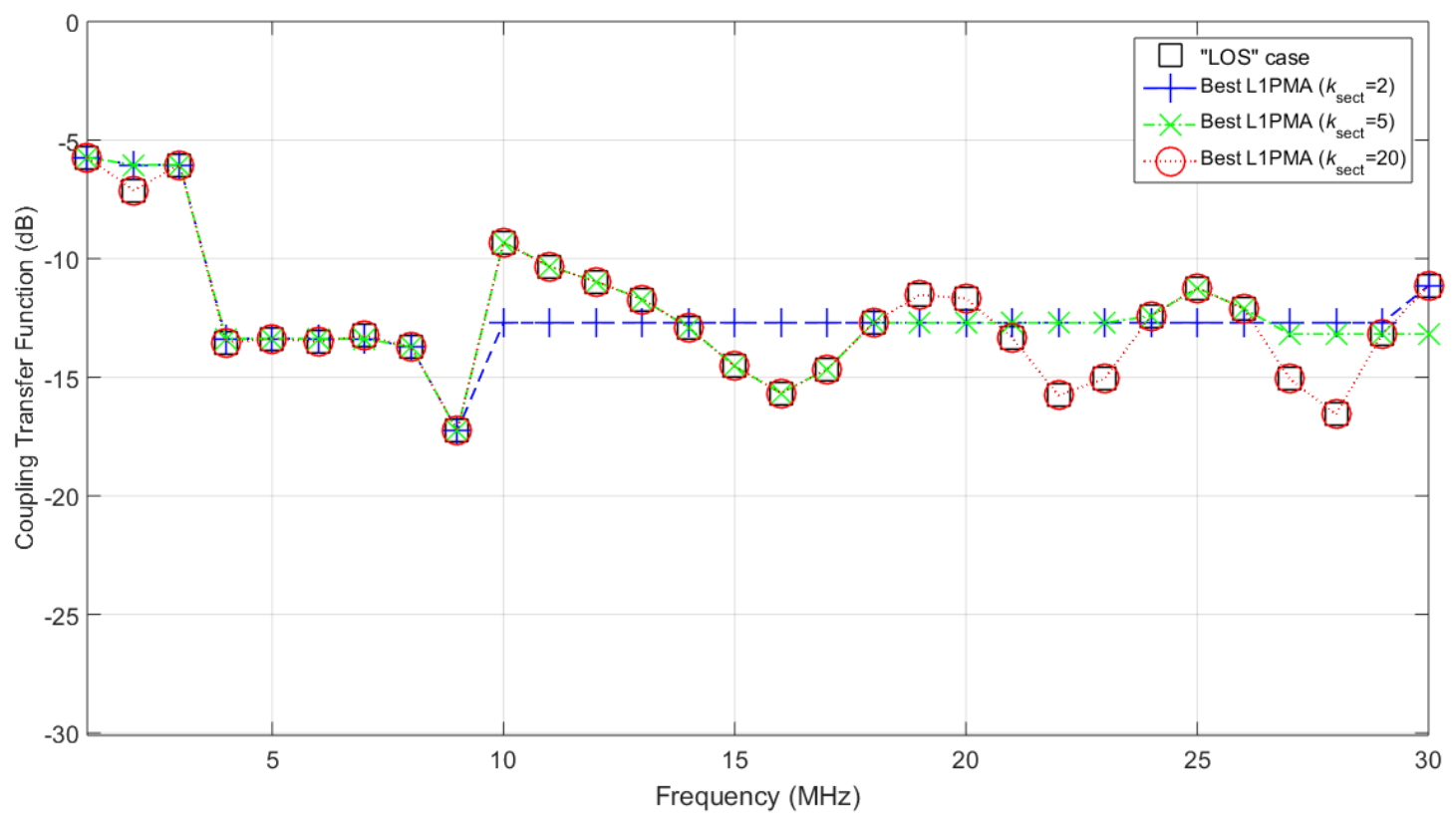

(c)

Figure 3. Theoretical and approximated coupling transfer functions of the three indicative topologies of OV HV BPL networks when best L1PMA is applied (a) Suburban case. (b) Rural case. (c) "LOS" case.

TABLE I

Average Errors between Theoretical and Approximated Coupling Transfer Functions

\begin{tabular}{|c|c|c|c|}
\hline \multirow{2}{*}{$\begin{array}{c}\text { Number of } \\
\text { Monotonic } \\
\text { Sections }\end{array}$} & \multicolumn{3}{|c|}{$\begin{array}{l}\text { Average Error } \\
\text { (dB) }\end{array}$} \\
\hline & Suburban case & Rural case & "LOS" case \\
\hline 1 & 0.08 & 0.29 & -0.01 \\
\hline 2 & -0.23 & 0.44 & 0.26 \\
\hline 3 & -0.01 & 0.23 & 0.35 \\
\hline 4 & -0.10 & 0.38 & 0.31 \\
\hline 5 & -0.05 & 0.37 & 0.28 \\
\hline 6 & -0.14 & 0.26 & 0.17 \\
\hline 7 & -0.14 & 0.26 & 0.15 \\
\hline 8 & -0.04 & 0.16 & 0.04 \\
\hline 9 & -0.04 & 0.16 & 0.04 \\
\hline 10 & 0.03 & 0.01 & $5.33 \times 10^{-3}$ \\
\hline 11 & 0.03 & 0.01 & $5.33 \times 10^{-3}$ \\
\hline 12 & 0.04 & $1.79 \times 10^{-3}$ & $4.26 \times 10^{-3}$ \\
\hline 13 & 0.04 & $1.79 \times 10^{-3}$ & $4.26 \times 10^{-3}$ \\
\hline 14 & 0.03 & $-2.26 \times 10^{-7}$ & $1.81 \times 10^{-7}$ \\
\hline 15 & 0.03 & $-2.26 \times 10^{-7}$ & $1.81 \times 10^{-7}$ \\
\hline 16 & $4.76 \times 10^{-7}$ & $-2.26 \times 10^{-7}$ & $1.81 \times 10^{-7}$ \\
\hline 17 & $4.79 \times 10^{-7}$ & $-2.26 \times 10^{-7}$ & $1.81 \times 10^{-7}$ \\
\hline 18 & $4.79 \times 10^{-7}$ & $-2.26 \times 10^{-7}$ & $1.81 \times 10^{-7}$ \\
\hline 19 & $4.79 \times 10^{-7}$ & $-2.26 \times 10^{-7}$ & $1.81 \times 10^{-7}$ \\
\hline 20 & $4.79 \times 10^{-7}$ & $-2.26 \times 10^{-7}$ & $1.81 \times 10^{-7}$ \\
\hline
\end{tabular}


Comparing Figs. 3(a)-(c) with Table 1, several interesting remarks can be pointed out, as follows:

- As already been observed in distribution BPL networks [1], best L1PMA may successfully approximate the coupling transfer functions in all the OV HV BPL topologies examined, namely:

○ "LOS" case is characterized by coupling transfer functions with few and shallow spectral notches. Due to the proximity of the conductors with reference to their height above the ground, significant cross-talk phenomena arise between the conductor couples of the same phase having as a result the presence of notches even during the "LOS" transmission case. Anyway, best L1PMA curves practically coincide with the "LOS" coupling transfer functions. In fact, only eight monotonic sections are required so that the coincidence clearly occurs. Note that the threshold of the coincidence is assumed equal to $0.1 \mathrm{~dB}$.

- Rural topologies are characterized by shallow and less-frequent spectral notches. Actually, because of their high branch length, OV HV BPL rural topologies act as power dividers of the "LOS" one, adding an approximately $3 \mathrm{~dB}$ decrease in their coupling transfer functions for each branch encountered [7]. Therefore, the poor multipath environment of both "LOS" and rural topologies allows to the best L1PMA to achieve an excellent approximation in both cases with low number of monotonic sections. Indeed, best L1PMA achieves to follow the curve from the first monotonic section while the coincidence occurs after ten monotonic sections.

- Suburban and urban topologies are characterized by deep and frequent spectral notches that pose a significant approximation challenge to the best L1PMA [1], [6]. This is due to the fact the branch length becomes short enough so that the channel attenuation of the different multipath paths during the BPL signal transmission become comparable, thus, creating a rich multipath environment. In fact, the spectral notches are added to the OV HV BPL coupling transfer functions of the "LOS" topology. Even in the bad case of the suburban topology, best L1PMA achieves to coincide with the OV HV coupling transfer functions after 16 monotonic sections.

- In contrast with wavelet or spline approximations [1], [49], [50], best L1PMA result successfully includes either the primary extrema or the tail of the OV HV coupling transfer functions.

With reference to Table 1, even from the first monotonic section, the best L1PMA achieves an average error that is equal to $0.08 \mathrm{~dB}, 0.29 \mathrm{~dB}$ and $-0.01 \mathrm{~dB}$ for the suburban, rural and "LOS" case, respectively. This indicates that best L1PMA identifies and follows the crucial areas of OV HV BPL coupling functions whose neglect significantly affects the average error. At the same time, best L1PMA disregards the frequent fluctuations whose contribution to the average error remains topically and low. In reality, this is the cornerstone of the TIM; the measurement differences are ignored by the best L1PMA while the curve essence of the OV HV BPL coupling transfer functions remains. 


\subsection{OV HV BPL Topology Database and Computational Load}

With reference to Sec. IIC, to create a detailed OV HV BPL topology database, all the possible OV HV BPL topologies concerning the number of branches, each branch distance from the transmitting end and each branch length need to be added in the database. Taking under consideration the OV HV BPL topology database specifications of Sec.VA, there is a need for inserting 20 approximated theoretical OV HV BPL coupling transfer function column vectors per each possible OV HV BPL topology of the database, which corresponds to the respective 20 monotonic sections. Note that each approximated theoretical OV HV BPL coupling transfer function column vector consists of 30 elements, which corresponds to the respective 30 measurement frequencies. Therefore, the detailed OV HV BPL topology database comprises:

- "LOS" transmission case: 1 topology and $1 \times 20=20$ corresponding approximated theoretical OV HV BPL coupling transfer function column vectors. In total, 600 elements are required to be inserted in the database.

- $O V H V$ BPL topologies with one branch: With reference to eq. (1), there are 676 topologies and 13,520 corresponding approximated theoretical OV HV BPL coupling transfer function column vectors. In total, 405,600 elements are required to be inserted in the database.

- OV HV BPL topologies with two branches: With reference to eq. (1), there are 237,276 topologies and 4,745,520 corresponding approximated theoretical OV HV BPL coupling transfer function column vectors. In total, 142,365,600 elements are required to be inserted in the database.

- $O V H V$ BPL topologies with $N$ branches: With reference to eq. (1), there are $\sum_{i_{1}=1}^{26}\left\{\sum_{i_{2}=1}^{i_{1}}\left\{\cdots \sum_{i_{N-1}=1}^{i_{N-2}}\left\{i_{N-1}\right\}\right\}\right\} \times 26^{N}$ and $\sum_{i_{1}=1}^{26}\left\{\sum_{i_{2}=1}^{i_{1}}\left\{\cdots \sum_{i_{N-1}=1}^{i_{N-2}}\left\{i_{N-1}\right\}\right\}\right\} \times 26^{N} \times 20$ corresponding approximated theoretical OV HV BPL coupling transfer function column vectors.

In total, $\sum_{i_{1}=1}^{26}\left\{\sum_{i_{2}=1}^{i_{1}}\left\{\ldots \sum_{i_{N-1}=1}^{i_{N-2}}\left\{i_{N-1}\right\}\right\}\right\} \times 26^{N} \times 600$ elements are required to be inserted in the database.

Practically, to create the OV HV BPL topology database, the computational load of only inserting the elements of the "LOS" transmission case and OV HV BPL topologies with one branch is equal to 406,200 records or 7,886s. This implies that each record approximately requires $0.0194 \mathrm{~s}$ so that both the hybrid method and the best L1PMA are applied and the element insertion is complete. In Table 2, the number of elements and the approximated time duration of inserting OV HV BPL topologies of different number of branches is reported. Note that as the technical characteristics of the system performing the simulations are concerned, it has an Intel Pentium $1.9 \mathrm{GHz} \mathrm{CPU}$ and 4GB RAM.

From Table 2, it is evident that the approximated time duration poses significant technical difficulties during the consideration of OV HV BPL topologies with high number of branches in the database. In fact, when the number of branches is equal or greater than four, the approximated time duration exceeds the year. These cases rarely occur in OV HV BPL topologies of average path length of $25 \mathrm{~km}$ and for that reason are 
TABLE II

Number of Elements and Approximated Time Duration of OV HV BPL Topologies

\begin{tabular}{|c|c|c|}
\hline Number of Branches & Number of Elements & $\begin{array}{c}\text { Approximated Time Duration } \\
\text { (hours) }\end{array}$ \\
\hline 0 & 600 & 0.003 \\
\hline 1 & 405,600 & 2.19 \\
\hline 2 & $142,365,600$ & 767.19 \\
\hline 3 & $3.45 \times 10^{10}$ & $1.86 \times 10^{5}$ \\
\hline 4 & $6.51 \times 10^{12}$ & $3.51 \times 10^{7}$ \\
\hline 5 & $1.2 \times 10^{15}$ & $5.47 \times 10^{9}$ \\
\hline
\end{tabular}

omitted in this paper. Anyway, as detailed in the Sec.IVH of [59], the optimization of the insertion methodology in OV HV BPL topology database is among the critical steps of the future research. In this paper, only the cases of "LOS" case and topologies with one branch are considered during the proposed TIM for the sake of simplicity and speed (see Sec.VE).

\subsection{OV HV BPL Coupling Transfer Functions and Measurement Differences}

As already been reported in Sec.IVB, there are six categories that can create significant measurement differences between measurements and theoretical results during the determination of OV HV BPL coupling transfer functions. Based on the hybrid method and best L1PMA, the theoretical OV HV BPL coupling transfer functions can be revealed even though significant measurement differences may occur. In fact, best L1PMA achieves to mitigate the additive measurement by simply maintaining the monotonicity pattern of each coupling transfer function, which is imposed by the number of monotonic sections. In accordance with [1], the total occurred fault due to the six measurement difference categories $e(\cdot)$, which is described in eq. (5), can be assumed to follow either continuous uniform distribution (CUD) with minimum value $-a_{\text {CUD }}$ and maximum value $a_{\mathrm{CUD}}$ or normal distribution (ND) with mean $m_{\mathrm{ND}}$ and standard deviation $S$ ND.

The impact of measurement differences on the OV HV BPL coupling transfer functions is investigated in the subsection. More specifically, in Figs. 4(a)-(c), the theoretical coupling transfer function is plotted versus frequency for the indicative OV HV BPL topologies, respectively. In each figure, the measured coupling transfer function is also given when measurement differences follow the indicative measurement difference distributions: (i) $\mathrm{CUD}$ with $a_{\mathrm{CUD}}=3 \mathrm{~dB}$; and (ii) $\mathrm{ND}$ with $m_{\mathrm{ND}}=0 \mathrm{~dB}$ and $s \mathrm{ND}=2 \mathrm{~dB}$. Moreover, the result of best L1PMA is also curved for the measured coupling transfer function when six monotonic sections are assumed. 


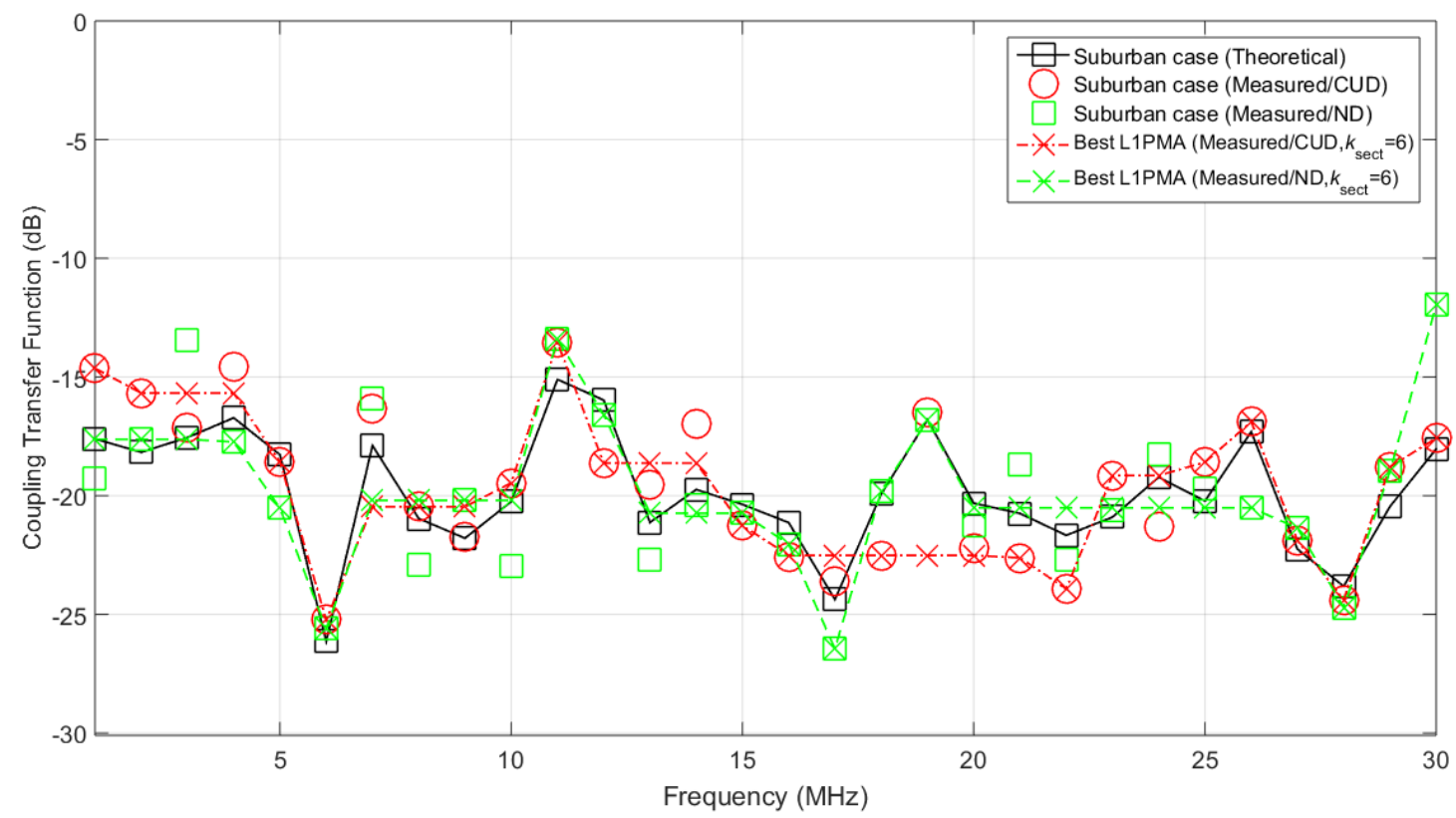

(a)

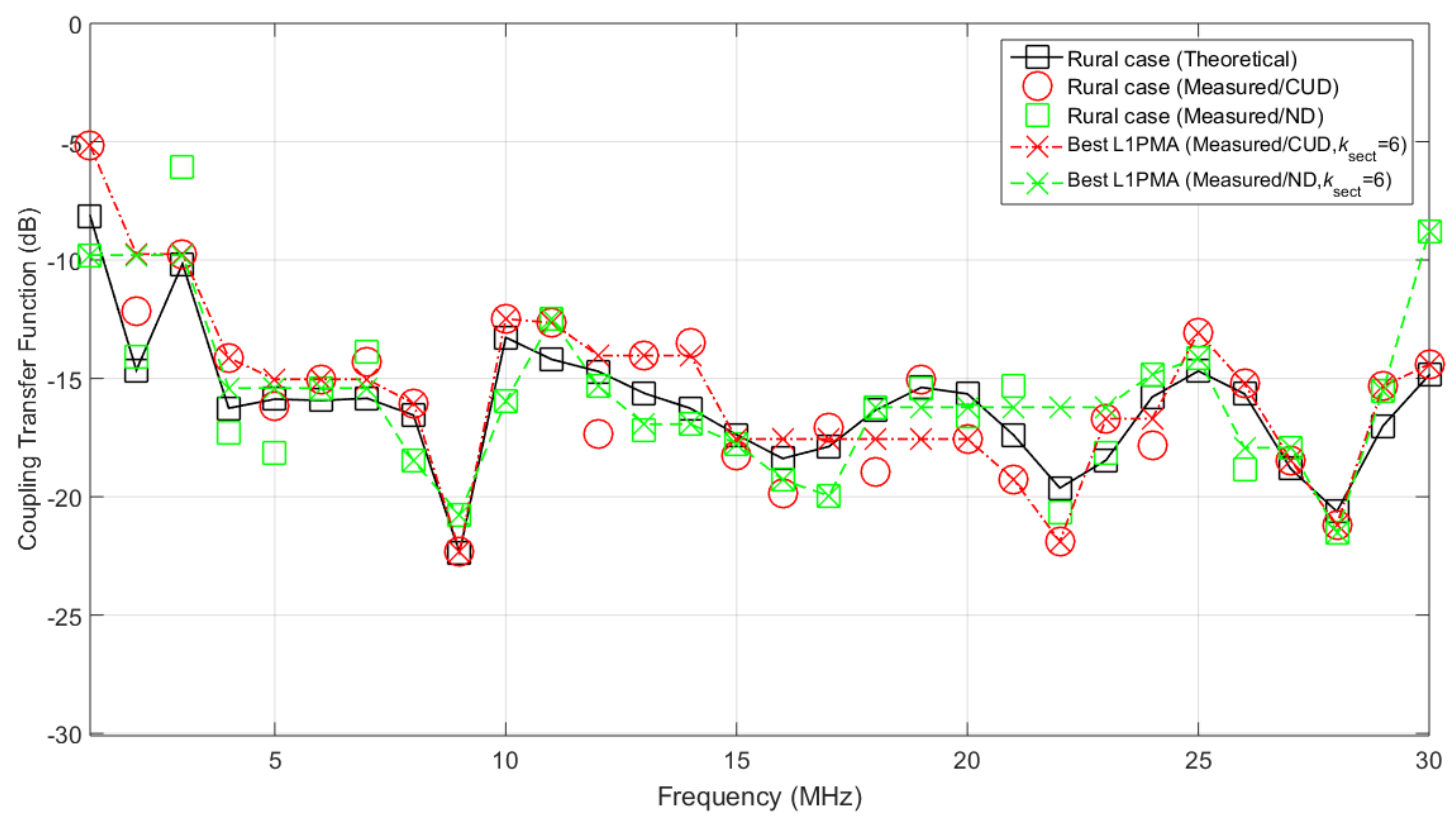

(b) 


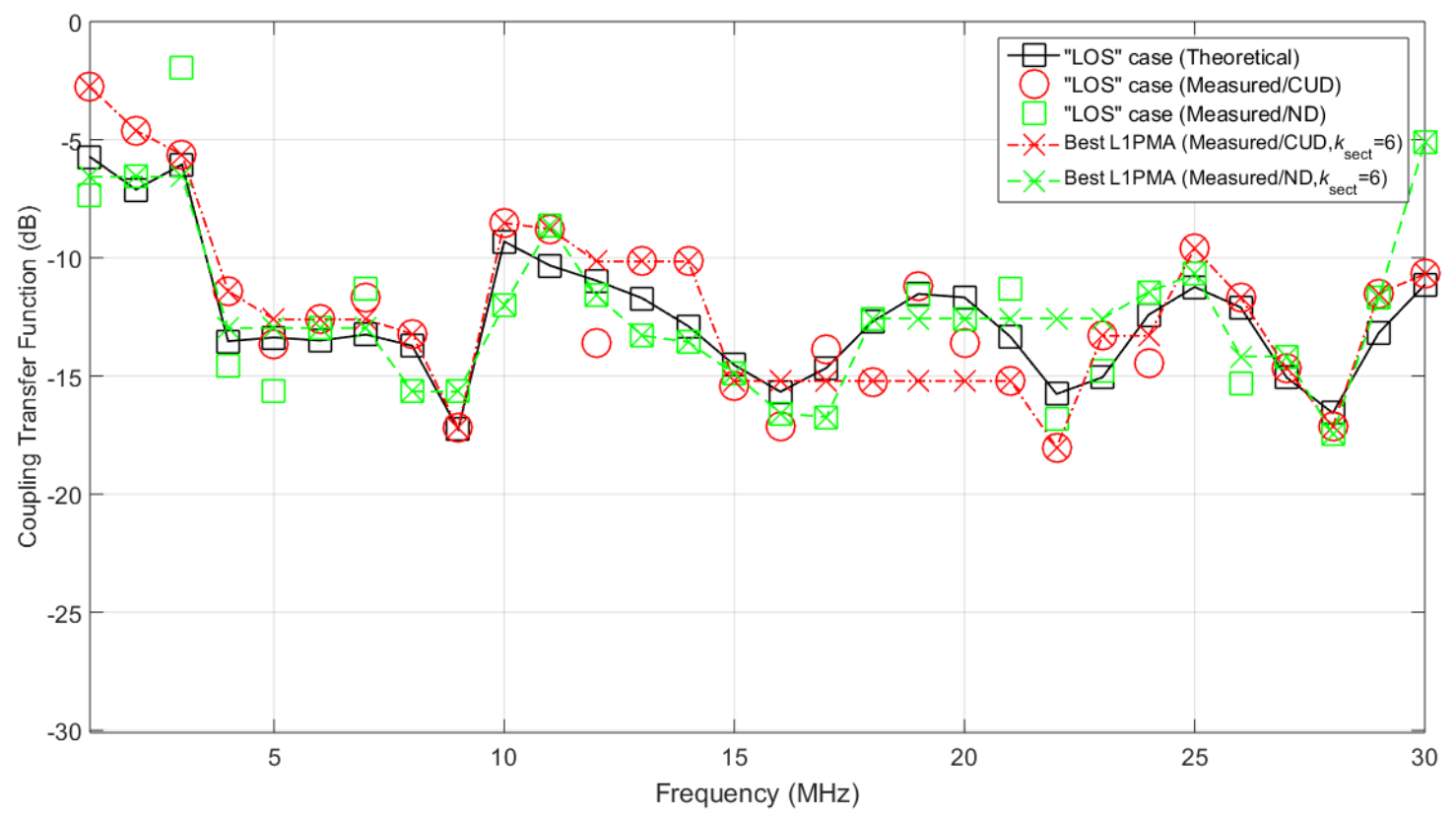

(c)

Figure 4. Theoretical, measured and approximated coupling transfer functions of the three indicative OV HV BPL topologies when best L1PMA is applied and two indicative fault distributions (CUD and ND) are adopted. (a) Suburban case. (b) Rural case. (c) "LOS" case.

From Figs. 3(a)-(c), it has been pointed out that best L1PMA first identifies the most important local extrema of the theoretical coupling transfer functions, which are treated as primary extrema. Then, best L1PMA interpolates the data at these extrema. The increase of the number of monotonic sections implies that the best L1PMA achieves to include even more secondary extrema in its results, thus, enhancing the accuracy of the approximation. From Figs. 4(a)-(c), it is obvious that the measurement differences, which may follow either CUD or ND, little affect the general form of the coupling transfer functions. Indeed, given the number of the monotonic sections, best L1PMA first tries to include the primary extrema of measured coupling transfer functions, which little differ from the respective primary extrema of the theoretical transfer functions, and second performs the best fit among the data in every monotonous section. In general terms, best L1PMA tries to restore the theoretical coupling transfer functions through its data smoothing capability.

\subsection{TIM and the Set of Candidate OV HV BPL Topologies}

As the restoration effectiveness of best L1PMA to theoretical and measured coupling transfer functions is well presented in the case of the indicative suburban, rural and "LOS" topologies, there are two significant drawbacks concerning the topology identification from the measured coupling transfer functions: (i) When a low number of monotonic sections is assumed, best L1PMA result follows the general trend of the respective measured coupling transfer function that resembles to the respective theoretical one but it loses significant primary extrema. (ii) When a high number of monotonic sections is assumed, best L1PMA result tends to identify both primary and 
secondary extrema but exaggerates to include them, thus, giving important role to the measurement differences during the approximations. Taking into account the previous two performance tips, during the proposed TIM: (a) The TIM test is going to be applied between the approximated theoretical and measured coupling transfer functions for the same number of monotonic sections in both cases. (b) To exploit the advantages of the different number of monotonic sections, the TIM test includes the entire range of the available monotonic sections.

With reference to Sec.IVF, the three steps of the proposed TIM are now detailed:

1. The computation of measured OV HV coupling transfer function column vector $\overline{\mathbf{H}^{\mathrm{WtG}}}(\mathbf{f})$ and the approximated OV HV coupling transfer function column vector

$\overline{\overline{\mathbf{H}_{\text {meas }}^{\mathrm{WtG}}}}\left(\mathbf{f}, k_{\text {sect }}\right)$ for different number of monotonic sections has been presented in Sec.VD.

2. With reference to eq. (13), the OV HV BPL topology database of Sec.VC and the approximated measured OV HV BPL coupling transfer functions of Sec.VD, the $C S P M_{\text {tot }}$ of the indicative topologies is calculated with respect to the topologies of the database. In Fig. 5(a), the CSPM tot of the indicative rural topology and its position among the $677 \mathrm{OV}$ HV BPL topologies of the database in ascending $C S P M$ tot order are plotted versus the CUD maximum value $a_{\mathrm{CUD}}$. Apart from the proposed TIM, the respective position of the traditional TIM is also demonstrated. Similar curves with Fig. 5(a) are given in Fig. 5(b) but for different values of ND standard deviation $s_{\mathrm{ND}}$ when $m_{\mathrm{ND}}$ is equal to 0dB. In Figs. 5(c) and 5(d), similar curves with Figs. 5(a) and 5(b) are presented but for the indicative "LOS" topology.

3. A set of candidate OV HV BPL topologies with their respective CSPM tot is provided by the TIM as it is shown in Figs. 5(a)-(d). All these topologies present the same $C S P M$ tot and coincide at the first position in ascending $C S P M$ tot order. 


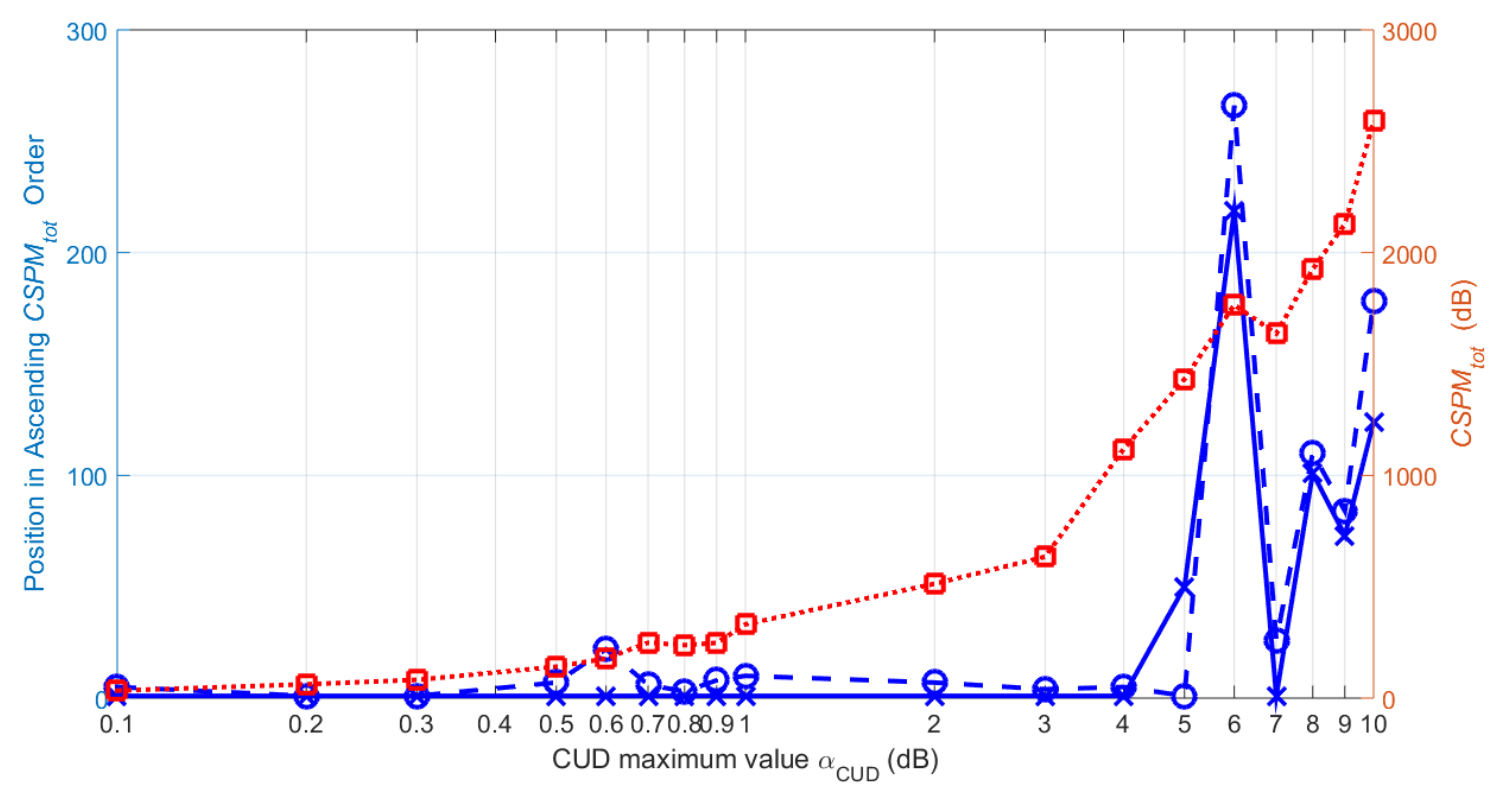

(a)

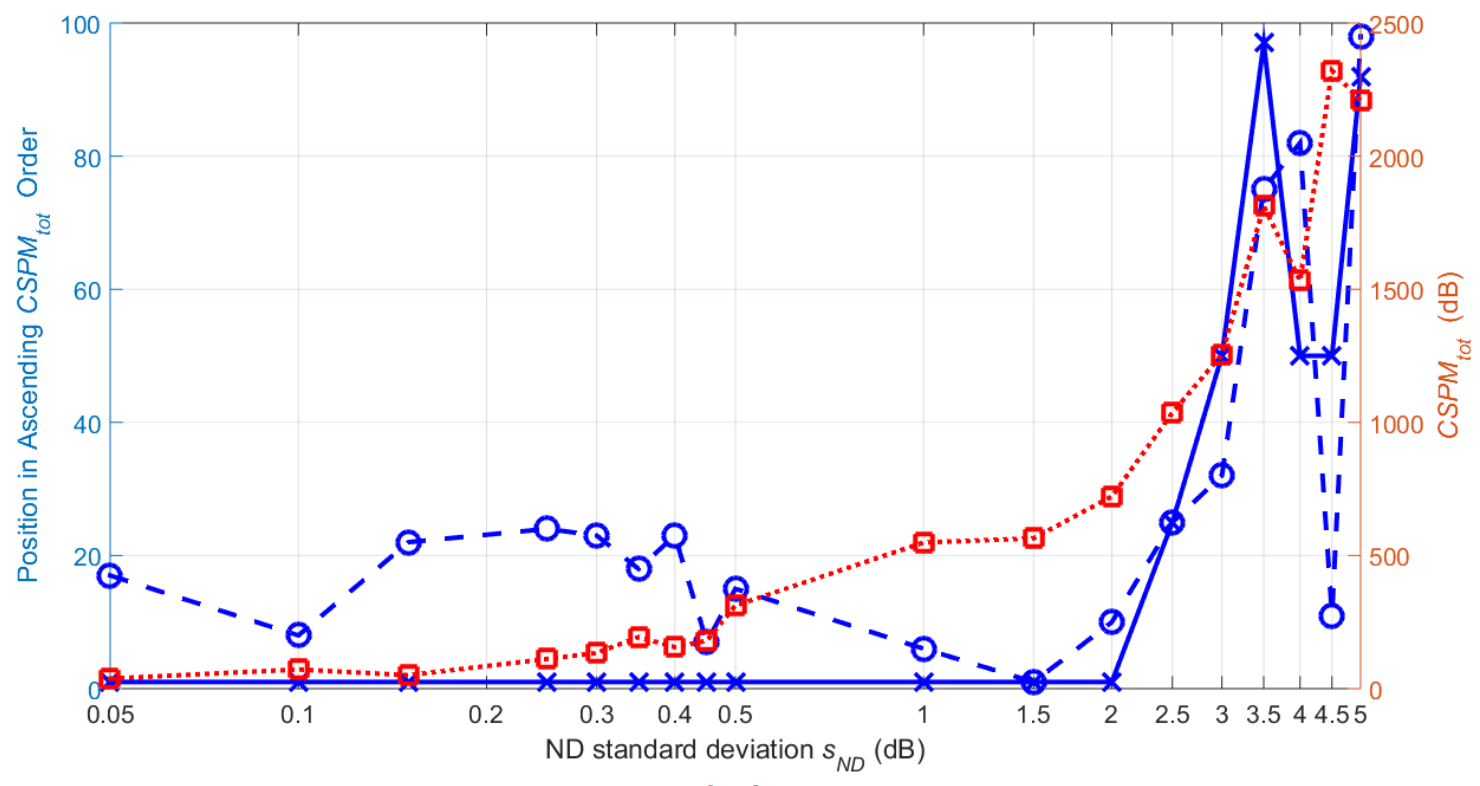

(b) 


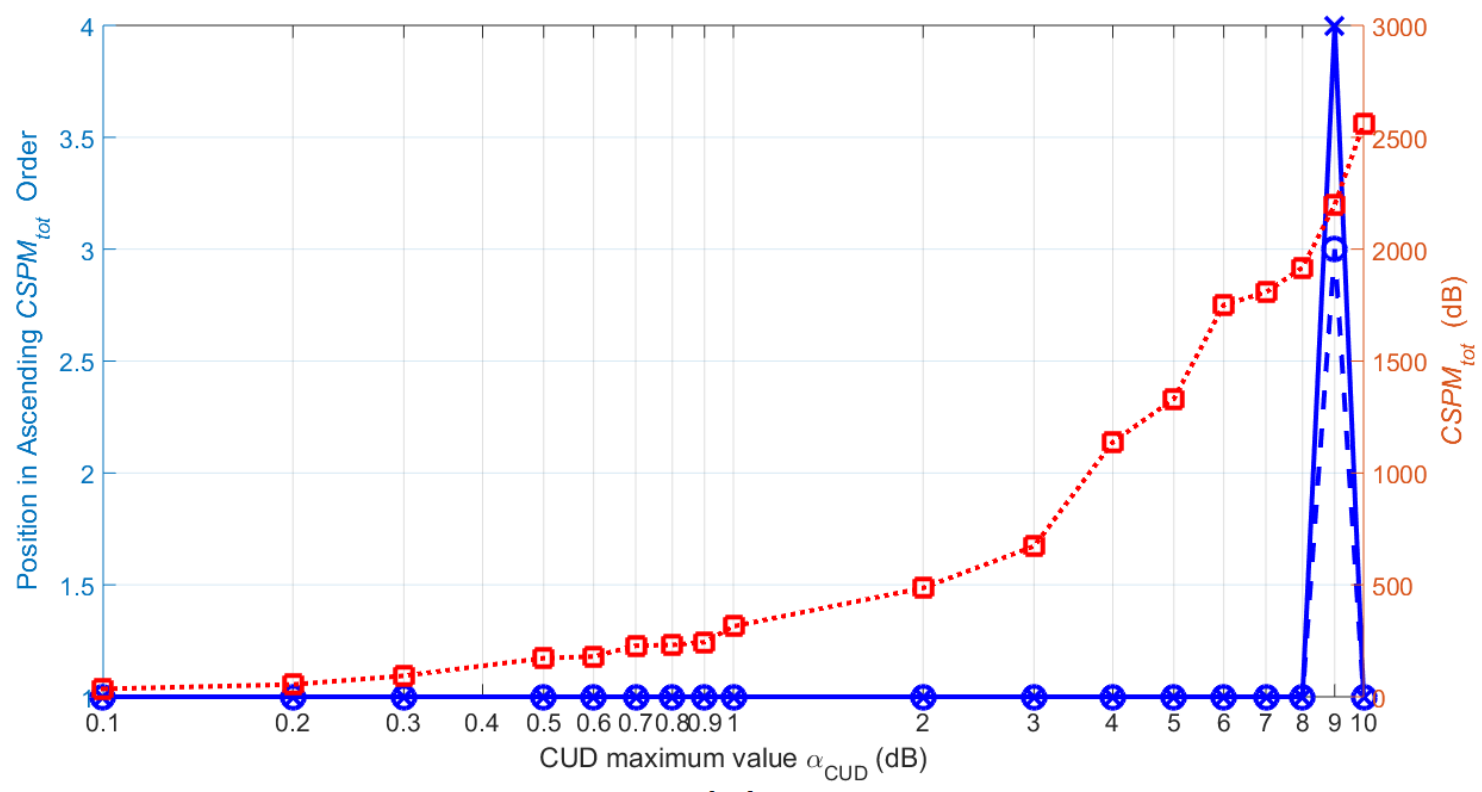

(c)

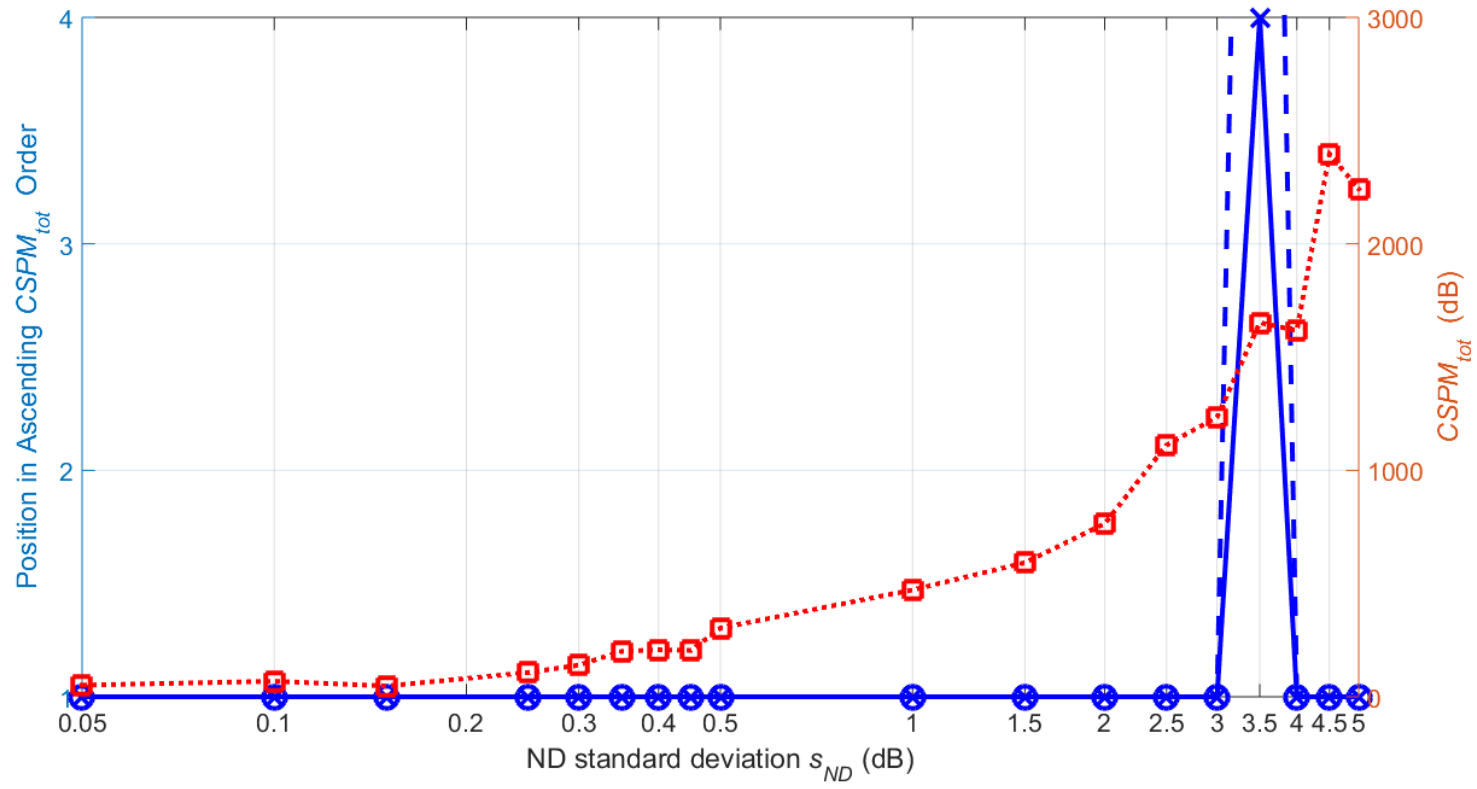

(d)

Figure 5. CSPMtot ( $\square$ ) and position in ascending CSPMtot order versus measurement difference distribution characteristics when traditional TIM (- $\left.{ }^{-}\right)$and proposed TIM $\left({ }^{-}{ }^{-}\right)$are applied. (a) CUD / Rural case. (b) ND / Rural case. (c) CUD / "LOS" case. (d) ND / "LOS" case.

Observing Figs. 5(a)-(d), several interesting remarks concerning the TIM can be pointed out:

- "LOS" topology presents slightly lower CSPM values in comparison with the indicative rural ones. This is an obvious result since the additive spectral notches of the indicative rural topology challenge the efficiency of the proposed TIM in 
contrast with the "LOS" case where its revelation from TIM is a straightforward procedure.

- The proposed TIM succeeds in identifying the underlaid OV HV BPL topology more precisely than traditional one in the vast majority of the cases examined. Actually, TIM successfully reveals the "LOS" and indicative rural topology even if respective critical measurement differences that may reach up to $10 \mathrm{~dB}$ of measurement difference magnitude occur.

- Apart from curve similarity metric and topology identification tool, CSPM value warns about its accuracy degree. In fact, higher values of CSPM imply that: (i) topology identification becomes more precarious due to the rich multipath environment of the underlaid OV HV BPL topology; (ii) the spectral notches that are superimposed by the measurement differences on the coupling transfer functions become comparable with the inherent ones. In fact, CSPM values increase as the magnitude of measurement differences increases too in all the cases examined; (iii) even if the position of the underlaid OV HV BPL topology is first in ascending $C S P M_{\text {tot }}$ order among the other database topologies, the number of candidate topologies that are characterized by the same $C S P M_{\text {tot }}$ with the underlaid one increases; and (iv) the $C S P M$ tot difference between the candidate OV HV BPL topologies of the set and the first topology outside the set decreases.

- The main component of TIM is its best L1PMA module. Based on the primary extrema, which are the main spectral notches, best L1PMA decomposes BPL transfer functions into separate monotonous sections between the primary extrema [1], [35], [36]. Since the number and the length of branches critically determine the number and the depth of spectral notches, the best L1PMA outputs of OV HV BPL topologies with the same branch number and length resemble each other regardless of the number of monotonic sections considered. This is verified by the same branch number and length that present the candidate OV HV BPL topologies of the set. Here, it is expected that the number of the candidate rural topologies is high since all the OV HV BPL topologies of one long branch resemble to the power divider behavior as described in Sec.VB.

- If the OV HV BPL topology is already known then the exact measurement differences can be computed on the basis of CSPM tot curves. Note that steep changes in the characteristics of the measurement difference distribution imply changes in the surrounding environment of OV HV BPL topologies.

Since the proposed TIM can identify an OV HV BPL topology even if measurement differences occur during the determination of its coupling transfer function, each OV HV BPL topology is almost uniquely characterized by the curve trend and the values of the CSPM tot. Since two OV HV BPL topologies present different CSPM tot behavior, these topologies can be differentiated from each other. This implies that various serious faults and instabilities across the transmission power grid (e.g., line faults and line termination instabilities) can be first differentiated from the measurement differences and, then, be identified for given OV HV BPL topology. In the companion paper of [59], the proposed TIM is extended to the Fault and Instability Identification Methodology (FIIM) so that faults or instabilities across an intelligent energy system can be identified and the personnel, who is monitoring the transmission power grid, is warned so as to restore the power grid to default settings. 


\section{Conclusions}

In this paper, the proposed TIM of an OV HV BPL topology, when a corresponding OV HV BPL coupling transfer function with measurement differences is available, has been presented. The TIM consists of: (i) the hybrid method; (ii) the best L1PMA; (iii) the proposed OV HV BPL topology database; and (iv) CSPM that serves as the assessment metric of the TIM accuracy.

As the validation of the TIM is concerned, the CSPM has achieved to reveal a set of candidate OV HV BPL topologies with the underlaid topology lying inside it even though measurement differences of various distributions and magnitudes have been applied.

In the companion paper of [59], TIM is extended to FIIM so that faults and instabilities across the OV HV BPL networks can be identified and the responsible personnel is warned.

\section{Conflicts of Interest}

The author declares that there is no conflict of interests regarding the publication of this paper.

\section{References}

[1] A. G. Lazaropoulos, "Best L1 Piecewise Monotonic Data Approximation in Overhead and Underground Medium-Voltage and Low-Voltage Broadband over Power Lines Networks: Theoretical and Practical Transfer Function Determination," Hindawi Journal of Computational Engineering, vol. 2016, Article ID 6762390, 24 pages, 2016. DOI: 10.1155/2016/6762390.

[2] C. Cano, A.Pittolo, D. Malone, L. Lampe, A. M.Tonello, and A. Dabak, "State-ofthe-art in Power Line Communications: From the Applications to the Medium," IEEE J. Sel. Areas Commun., vol. 34, pp. 1935-1952, 2016.

[3] A. Milioudis, G. Andreou, and D. Labridis, "Optimum transmitted power spectral distribution for broadband power line communication systems considering electromagnetic emissions," Elsevier Electric Power Systems Research, vol. 140, pp. 958-964, 2016. DOI: 10.1016/j.epsr.2016.03.047

[4] T. A. Papadopoulos, A. I. Chrysochos, E. O. Kontis, and G. K. Papagiannis, "Ringdown Analysis of Power Systems Using Vector Fitting," Electric Power Systems Research, vol. 141, pp. 100-103, 2016.

[5] A. G. Lazaropoulos, "Broadband transmission characteristics of overhead highvoltage power line communication channels," Progress in Electromagnetics Research B, vol. 36, pp. 373-398, 2012. [Online]. Available: http://www.jpier.org/PIERB/pierb36/19.11091408.pdf

[6] A. G. Lazaropoulos, "Broadband transmission and statistical performance properties of overhead high-voltage transmission networks," Hindawi Journal of Computer Networks and Commun., 2012, article ID 875632, 2012. [Online]. Available: http://www.hindawi.com/journals/jcnc/aip/875632/

[7] A. G. Lazaropoulos, "Review and Progress towards the Common Broadband Management of High-Voltage Transmission Grids: Model Expansion and Comparative Modal Analysis," ISRN Electronics, vol. 2012, Article ID 935286, pp. $1-18,2012 . \quad$ [Online]. Available: http://www.hindawi.com/isrn/electronics/2012/935286/ 
[8] A. G. Lazaropoulos, "Capacity Performance of Overhead Transmission MultipleInput Multiple-Output Broadband over Power Lines Networks: The Insidious Effect of Noise and the Role of Noise Models.," Trends in Renewable Energy, vol. 2, no. 2, pp. 61-82, Jan. 2016. DOI: 10.17737/tre.2016.2.2.0023

[9] A. I. Chrysochos, T. A. Papadopoulos, A. ElSamadouny, G. K. Papagiannis, and N. Al-Dhahir, "Optimized MIMO-OFDM design for narrowband-PLC applications in medium-voltage smart distribution grids," Electric Power Systems Research, vol. 140, pp. 253-262, 2016. DOI: 10.1016/j.epsr.2016.06.017

[10] A. G. Lazaropoulos and P. Lazaropoulos, "Financially Stimulating Local Economies by Exploiting Communities' Microgrids: Power Trading and Hybrid Techno-Economic (HTE) Model," Trends in Renewable Energy, vol. 1, no. 3, pp. 131-184, Sep. 2015. DOI: 10.17737/tre.2015.1.3.0014

[11] A. G. Lazaropoulos, "Wireless Sensor Network Design for Transmission Line Monitoring, Metering and Controlling: Introducing Broadband over PowerLinesenhanced Network Model (BPLeNM)," ISRN Power Engineering, vol. 2014, Article ID 894628, 22 pages, 2014. DOI:10.1155/2014/894628

[12] A. G. Lazaropoulos, "Wireless Sensors and Broadband over PowerLines Networks: The Performance of Broadband over PowerLines-enhanced Network Model (BPLeNM) (Invited Paper)," ICAS Publishing Group Transaction on IoT and Cloud Computing, vol. 2, no. 3, pp. 1-35, 2014. [Online]. Available: http://icas-pub.org/ojs/index.php/ticc/article/view/27/17

[13] S. S. Pappas, L. Ekonomou, D. C. Karamousantas, G. E. Chatzarakis, S. K. Katsikas, and P. Liatsis, "Electricity Demand Loads Modeling Using AutoRegressive Moving Average (ARMA) Models," Energy, vol. 33, no. 9, pp. 1353-1360, 2008. DOI: 10.1016/j.energy.2008.05.008

[14] A. G. Lazaropoulos and P. G. Cottis, "Transmission characteristics of overhead medium voltage power line communication channels," IEEE Trans. Power Del., vol. 24, no. 3, pp. 1164-1173, Jul. 2009.

[15] A. G. Lazaropoulos and P. G. Cottis, "Capacity of overhead medium voltage power line communication channels," IEEE Trans. Power Del., vol. 25, no. 2, pp. 723-733, Apr. 2010.

[16] A. G. Lazaropoulos and P. G. Cottis, "Broadband transmission via underground medium-voltage power lines-Part I: transmission characteristics," IEEE Trans. Power Del., vol. 25, no. 4, pp. 2414-2424, Oct. 2010.

[17] A. G. Lazaropoulos and P. G. Cottis, "Broadband transmission via underground medium-voltage power lines-Part II: capacity," IEEE Trans. Power Del., vol. 25, no. 4, pp. 2425-2434, Oct. 2010.

[18] F. Versolatto and A. M. Tonello, "An MTL theory approach for the simulation of MIMO power-line communication channels," IEEE Trans. Power Del., vol. 26, no. 3, pp. 1710-1717, Jul. 2011.

[19] M. Zimmermann and K. Dostert, "Analysis and modeling of impulsive noise in broad-band powerline communications," IEEE Trans. Electromagn. Compat., vol. 44, no. 1, pp. 249-258, Feb. 2002.

[20] A. G. Lazaropoulos, "Factors Influencing Broadband Transmission Characteristics of Underground Low-Voltage Distribution Networks," IET Commun., vol. 6, no. 17, pp. 2886-2893, Nov. 2012.

[21] A. G. Lazaropoulos, "Towards broadband over power lines systems integration: Transmission characteristics of underground low-voltage distribution power lines," 
Progress in Electromagnetics Research B, 39, pp. 89-114, 2012. [Online]. Available: http://www.jpier.org/PIERB/pierb39/05.12012409.pdf

[22] A. G. Lazaropoulos, "Towards modal integration of overhead and underground low-voltage and medium-voltage power line communication channels in the smart grid landscape: model expansion, broadband signal transmission characteristics, and statistical performance metrics (Invited Paper)," ISRN Signal Processing, in press, [Online]. Available: http://www.isrn.com/journals/sp/aip/121628/

[23] A. G. Lazaropoulos, "Review and Progress towards the Capacity Boost of Overhead and Underground Medium-Voltage and Low-Voltage Broadband over Power Lines Networks: Cooperative Communications through Two- and ThreeHop Repeater Systems," ISRN Electronics, vol. 2013, Article ID 472190, pp. 1-19, 2013. [Online]. Available: http://www.hindawi.com/isrn/electronics/aip/472190/

[24] A. G. Lazaropoulos, "Green Overhead and Underground Multiple-Input MultipleOutput Medium Voltage Broadband over Power Lines Networks: EnergyEfficient Power Control," Springer Journal of Global Optimization, vol. 2012 / Print ISSN 0925-5001, pp. 1-28, Oct. 2012.

[25] P. Amirshahi and M. Kavehrad, "High-frequency characteristics of overhead multiconductor power lines for broadband communications," IEEE J. Sel. Areas Commun., vol. 24, no. 7, pp. 1292-1303, Jul. 2006.

[26] T. Sartenaer, "Multiuser communications over frequency selective wired channels and applications to the powerline access network" Ph.D. dissertation, Univ. Catholique Louvain, Louvain-la-Neuve, Belgium, Sep. 2004.

[27] T. Calliacoudas and F. Issa, "“Multiconductor transmission lines and cables solver," An efficient simulation tool for plc channel networks development," presented at the IEEE Int. Conf. Power Line Communications and Its Applications, Athens, Greece, Mar. 2002.

[28] A. G. Lazaropoulos, "Policies for Carbon Energy Footprint Reduction of Overhead Multiple-Input Multiple-Output High Voltage Broadband over Power Lines Networks," Trends in Renewable Energy, vol. 1, no. 2, pp. 87-118, Jun. 2015. DOI: 10.17737/tre.2015.1.2.0011

[29] T. Sartenaer and P. Delogne, "Deterministic modelling of the (Shielded) outdoor powerline channel based on the multiconductor transmission line equations," IEEE J. Sel. Areas Commun., vol. 24, no. 7, pp. 1277-1291, Jul. 2006.

[30] C. R. Paul, Analysis of Multiconductor Transmission Lines. New York: Wiley, 1994.

[31] T. A. Papadopoulos, A. I. Chrysochos, and G. K. Papagiannis, "Narrowband Power Line Communication:Medium Voltage Cable Modeling and Laboratory Experimental Results,"Electric Power Systems Research, vol. 102, pp. 50-60, 2013.

[32] A. N. Milioudis, G. T. Andreou, and D. P. Labridis, "Enhanced Protection Scheme for Smart Grids Using Power Line Communications Techniques-Part II: Location ofHigh Impedance Fault Position," IEEE Trans. on Smart Grid, no. 3, vol. 4, pp. 1631-1640, 2012.

[33] I. C. Demetriou and M. J. D. Powell, "Least squares smoothing of univariate data to achieve piecewise monotonicity," IMA J. of Numerical Analysis, vol. 11, pp. 411-432, 1991.

[34] I. C. Demetriou and V. Koutoulidis“On Signal Restoration by Piecewise Monotonic Approximation", in Lecture Notes in Engineering and Computer 
Science: Proceedings of The World Congress on Engineering 2013,London, U.K., Jul. 2013, pp. 268-273.

[35] I. C. Demetriou, "An application of best $L 1$ piecewise monotonic data approximation to signal restoration," IAENG International Journal of Applied Mathematics, vol. 53, no. 4, pp. 226-232, 2013.

[36] I. C. Demetriou, "L1PMA: A Fortran 77 Package for Best L1 Piecewise Monotonic Data Smoothing," Computer Physics Communications, vol. 151, no. 1, pp. 315-338, 2003.

[37] I. C. Demetriou, "Data Smoothing by Piecewise Monotonic Divided Differences," Ph.D. Dissertation, Department of Applied Mathematics and Theoretical Physics, University of Cambridge, Cambridge, 1985.

[38] I. C. Demetriou, "Best L1 Piecewise Monotonic Data Modelling,"Int. Trans. Opl Res., vol. 1, no. 1, pp. 85-94,1994.

[39] I.C. Demetriou, "L1PMA: a Fortran 77 package for best L1 piecewise monotonic data smoothing," 2003 http://cpc.cs.qub.ac.uk/summaries/ADRF

[40] P. Amirshahi, "Broadband access and home networking through powerline networks" Ph.D. dissertation, Pennsylvania State Univ., University Park, PA, May 2006. [Online]. Available: http://etda.libraries.psu.edu/theses/approved/WorldWideIndex/ETD$\underline{\text { 1205/index.html }}$

[41] N. Suljanović, A. Mujčić, M. Zajc, and J. F. Tasič, "Approximate computation of high-frequency characteristics for power line with horizontal disposition and middle-phase to ground coupling," Elsevier Electr. Power Syst. Res., vol. 69, pp. 17-24, Jan. 2004.

[42] M. D'Amore and M. S. Sarto, "Simulation models of a dissipative transmission line above a lossy ground for a wide-frequency range-Part I: Single conductor configuration," IEEE Trans. Electromagn. Compat., vol. 38, no. 2, pp. 127-138, May 1996.

[43] M. D'Amore and M. S. Sarto, "Simulation models of a dissipative transmission line above a lossy ground for a wide-frequency range-Part II: Multi-conductor configuration," IEEE Trans. Electromagn. Compat., vol. 38, no. 2, pp. 139-149, May 1996.

[44] A. G. Lazaropoulos, "The Impact of Noise Models on Capacity Performance of Distribution Broadband over Power Lines (BPL) Networks," Hindawi Computer Networks and Communications, vol. 2016, Article ID 5680850, 14 pages, 2016. doi:10.1155/2016/5680850. [Online]. Available: http://www.hindawi.com/journals/jenc/2016/5680850/

[45] A. G. Lazaropoulos, "Designing Broadband over Power Lines Networks Using the Techno-Economic Pedagogical (TEP) Method - Part I: Overhead High Voltage Networks and Their Capacity Characteristics," Trends in Renewable Energy, vol. 1, no. 1, pp. 16-42, Mar. 2015. DOI: 10.17737/tre.2015.1.1.002

[46] OPERA1, D5: Pathloss as a function of frequency, distance and network topology for various LV and MV European powerline networks. IST Integrated Project No 507667, Apr. 2005.

[47] OPERA1, D44: Report presenting the architecture of plc system, the electricity network topologies, the operating modes and the equipment over which PLC access system will be installed, IST Integr. Project No 507667, Dec. 2005. 
[48] R. Pighi and R. Raheli, "On Multicarrier Signal Transmission for High-Voltage Power Lines," in Proc. IEEE Int. Symp. Power Line Commun. Appl., Vancouver, BC, Canada, Apr. 2005, pp. 32-36.

[49] C. de Boor, A Practical Guide to Splines. Revised Edition, NY: Springer-Verlag, Applied Mathematical Sciences, vol. 27, 2001.

[50] M. Holschneider, Wavelets. An Analysis Tool, Oxford: Clarendon Press, 1997.

[51] M. J. D. Powell, Approximation Theory and Methods. Cambridge, U.K.: Cambridge University Press, 1981.

[52] A. G. Lazaropoulos, Engineering the Art through the Lens of L1PMA: A Tribute to the Modern Greek Painters, Art Book ISSUU Digital Publishing Platform, Oct. 2014. [Online]. Available: http://issuu.com/lazaropoulos/docs/11pma

[53] A. G. Lazaropoulos, ReEngineering the Art through the Lens of L2WPMA: A Tribute to Leonardo da Vinci'sInventions: Flying Machines, War Machines, Architect/Innovations and Water Land Machines, Art Book ISSUU Digital Publishing Platform, Nov. 2014. [Online]. Available: http://issuu.com/lazaropoulos/docs/12wpma

[54] A. G. Lazaropoulos, ReEngineering the Art through the Lens of L1PMA L2WPMA: The Eternal Youth ofthe Parthenon Art, Architecture, Marble Sculpture, Metallurgy Pottery, Art Book ISSUU Digital Publishing Platform, Dec. 2014. [Online]. Available: http://issuu.com/lazaropoulos/docs/11pma 12wpma

[55] T. Banwell and S. Galli, "A novel approach to accurate modeling of the indoor power line channel-Part I: Circuit analysis and companion model," IEEE Trans. Power Del., vol. 20, no. 2, pp. 655-663, Apr. 2005.

[56] S. Galli and T. Banwell, "A novel approach to accurate modeling of the indoor power line channel - Part II: Transfer function and channel properties," IEEE Trans. Power Del., vol. 20, no. 3, pp. 1869-1878, Jul. 2005.

[57] S. Galli and T. Banwell, "A deterministic frequency-domain model for the indoor power line transfer function," IEEE J. Sel. Areas Commun., vol. 24, no. 7, pp. 1304-1316, Jul. 2006.

[58] A. G. Lazaropoulos, "Deployment Concepts for Overhead High Voltage Broadband over Power Lines Connections with Two-Hop Repeater System: Capacity Countermeasures against Aggravated Topologies and High Noise Environments," Progress in Electromagnetics Research B, vol. 44, pp. 283-307, 2012. [Online]. Available: http://www.jpier.org/PIERB/pierb44/13.12081104.pdf

[59] A. G. Lazaropoulos, "Measurement Differences, Faults and Instabilities in Intelligent Energy Systems - Part 2: Fault and Instability Prediction in Overhead High-Voltage Broadband over Power Lines Networks by Applying Fault and Instability Identification Methodology (FIIM)," Trends in Renewable Energy, 2(3), 113-142. DOI: 10.17737/tre.2016.2.3.0027

Article copyright: (C) 2016 Athanasios G. Lazaropoulos. This is an open access article distributed under the terms of the Creative Commons Attribution 4.0 International License, which permits unrestricted use and distribution provided the original author and source are credited. 